\title{
Effect of Prenatal Stress on Offspring's Learning, Memory and Coping Strategies
}

\section{Fatemeh Aghighi, Mahmoud Salami, Sayyed Alireza Talaei*}

Physiology Research Center, Institute for Basic Sciences, Kashan University of Medical Sciences, Kashan, Iran

\section{Article Info:}

\section{ABSTRACT}

Introduction: The prenatal stress is a key factor which affects the growth and function of the brain. Several studies have shown that prenatal stress induces deficits in learning and memory of the offspring. The prenatal stress alters the activity of neurotransmitters, such as noradrenaline, dopamine and serotonin, via over-activation of the hypothalamic-pituitaryadrenal axis. In addition, the prenatal stress reduces the values of various hippocampal proteins involved in learning and memory and long-term potentiation, like brain-derived neurotrophic factor, $\mathrm{Ca} 2+/$ calmodulin dependent protein kinase II, postsynaptic density protein 95, and cAMP response element-binding protein. It seems that exercise, environmental enrichment, and antioxidants could improve learning and memory deficits induced by the prenatal stress in offspring. Conclusion: Interventions to protect the offspring against stress is crucial to preserve their cognitive abilities.

Key words:

1. Maternal Exposure

2. Learning

3. Memory

4. Neuronal Plasticity

*Corresponding Author: Sayyed Alireza Talaei

E-mail: talaei@kaums.ac.ir 


\title{
تاثير استرس دوران باردارى بر حافظه و يادكيرى فرزندان و راهكارهاى مقابله با آن
}

\author{
فاطمه عقيقى، محمود سلامى، سيد عليرضا طلائى" \\ مركز تحقيقات فيزيولوزى، يزوهشكده علوم پايه، دانشعاه علوم يزشكى كاشان، كاشان، ايران
}

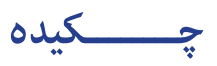

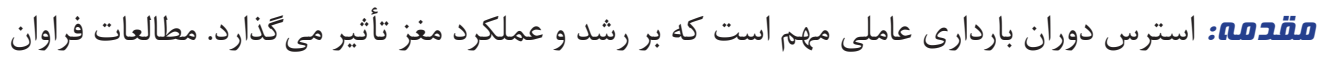

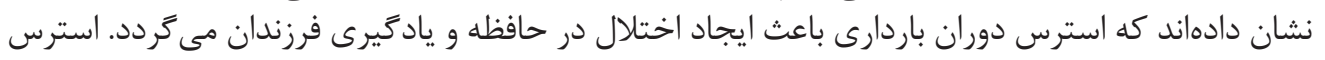

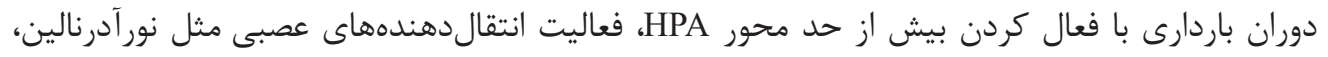

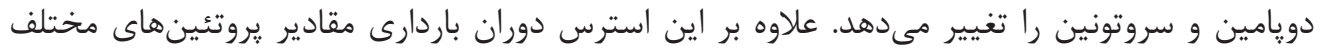

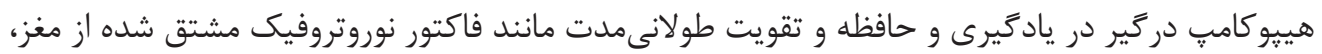

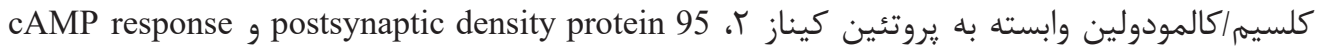

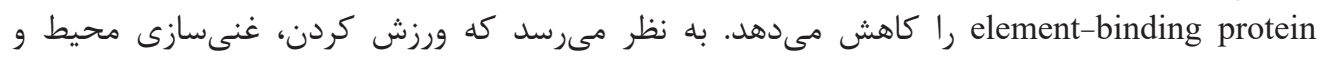

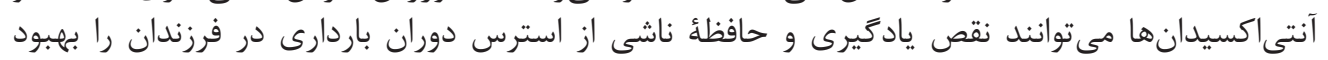

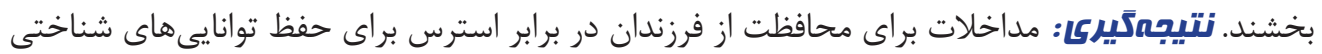
آنها بسيار مهمم است.

كليد وازهها:

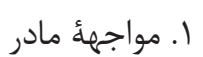

r. باد مافيرى

r. r. بافظه

ז

$$
\text { " نويسنده مسئول: سيد عليرضا طلائى : }
$$

آدرس الكترونيكى: talaei@kaums.ac.ir 


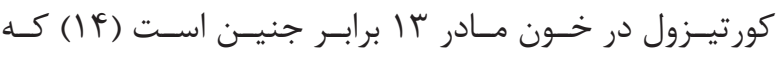

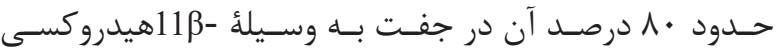

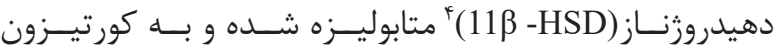

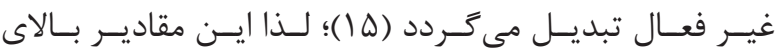

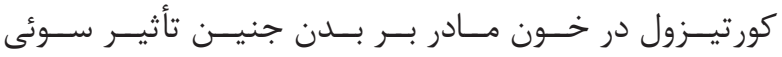

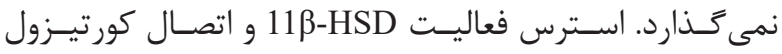

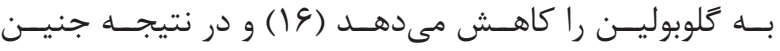

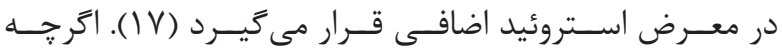

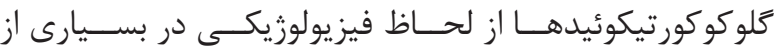

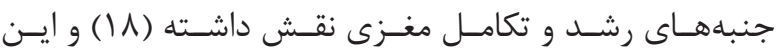

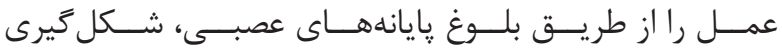

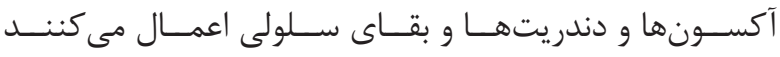

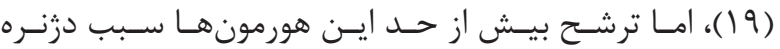

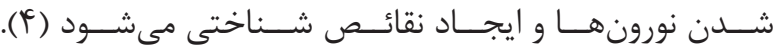

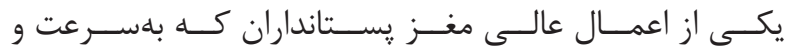

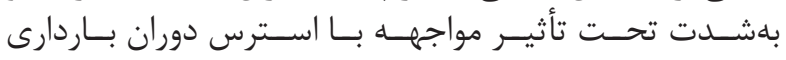

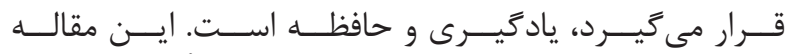

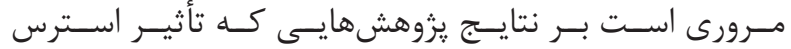

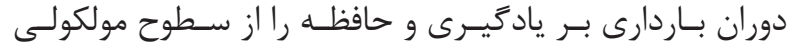

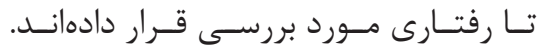

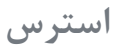

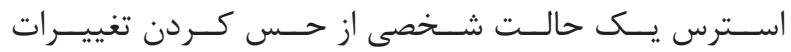

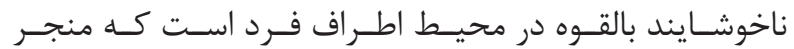

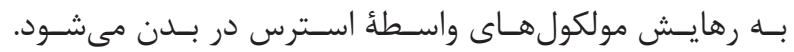

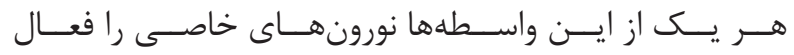

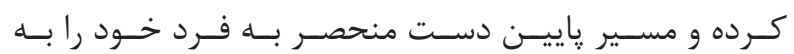

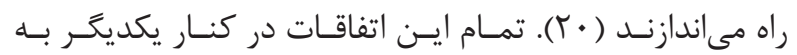

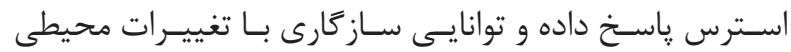

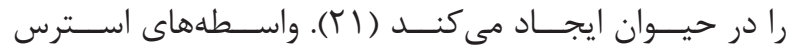

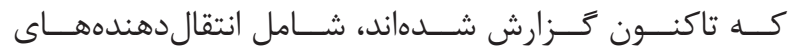

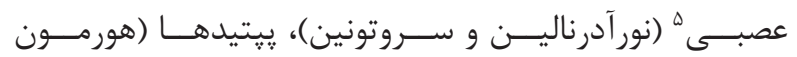

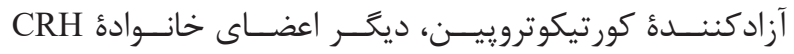

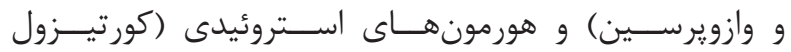

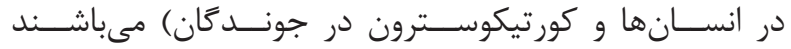

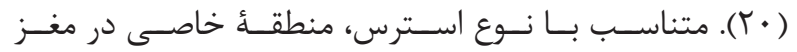

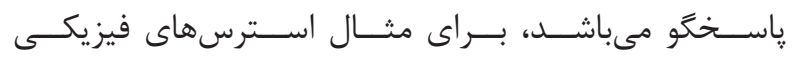

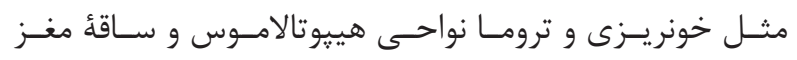

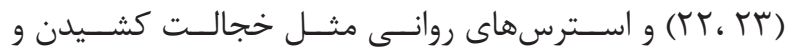

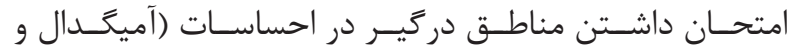

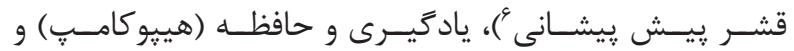

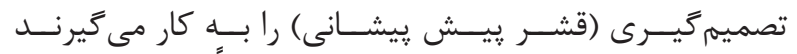

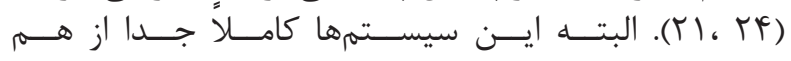

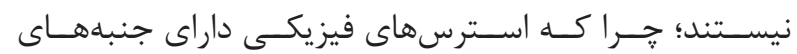

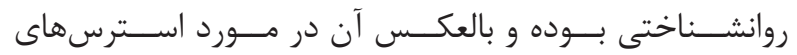

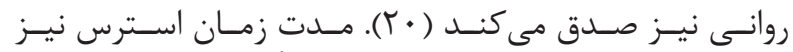

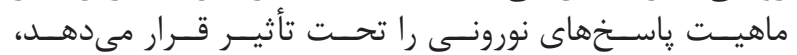

\footnotetext{
${ }^{1}$ Hypothalamus pituitary adrenal

${ }^{2}$ Prenatal stress

${ }^{3}$ Corticotropin-releasing hormone
}

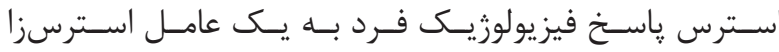

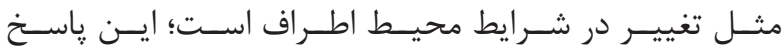

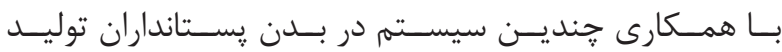

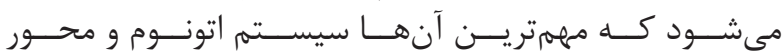

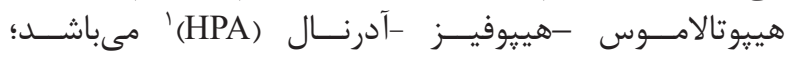

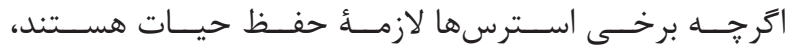

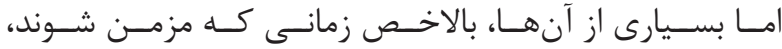

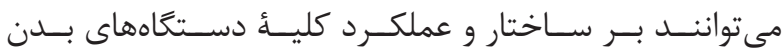

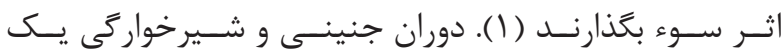

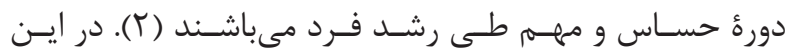

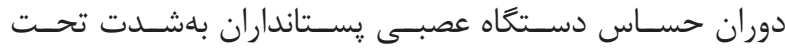

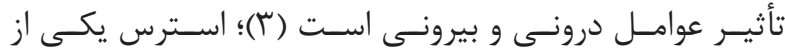

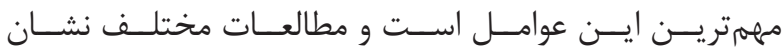

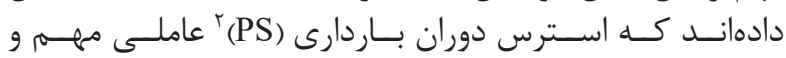

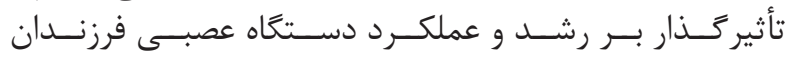

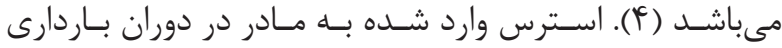

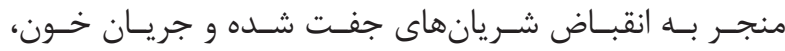

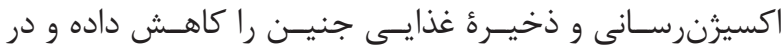

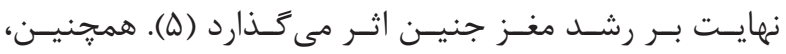

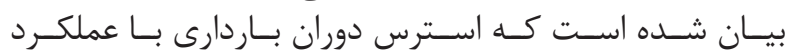

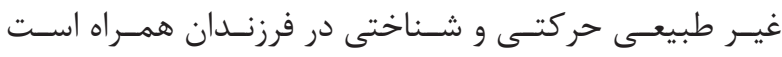
(Y، V)

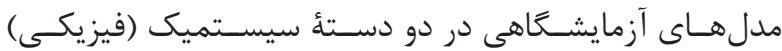

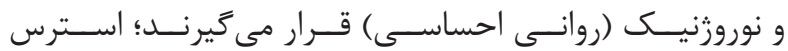

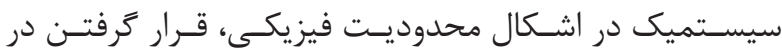

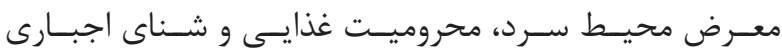

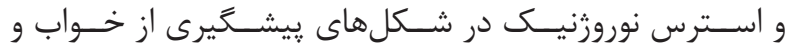

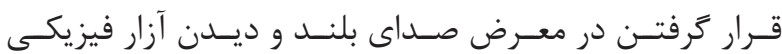

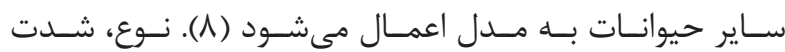

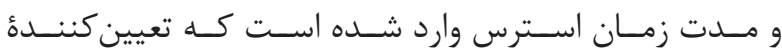

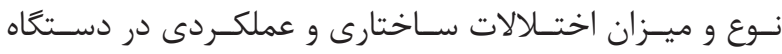

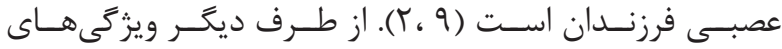

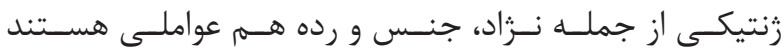

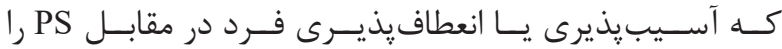

تعييـن مى كننـــــ (•) (1).

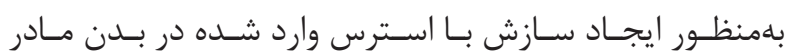

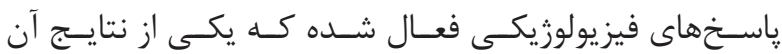

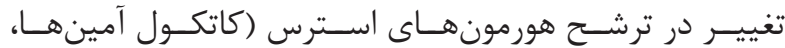

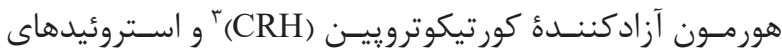

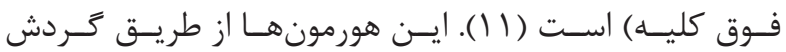

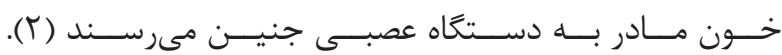

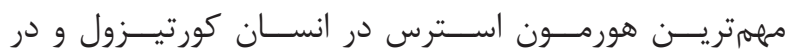

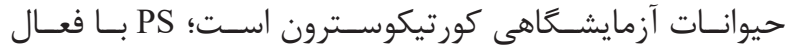

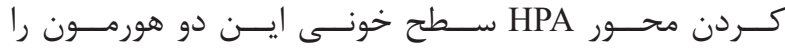

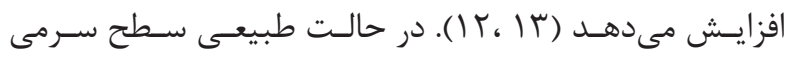

\footnotetext{
${ }^{4} 11 \beta$-hydroxysteroid dehydrogenase

${ }^{5}$ Neurotransmitters

${ }^{6}$ Prefrontal cortex
} 


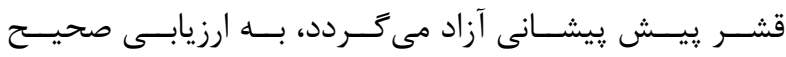

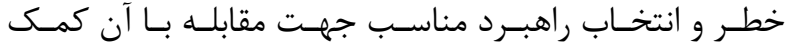

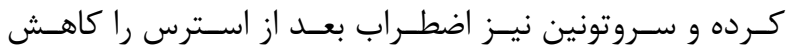

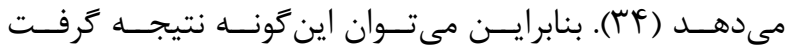

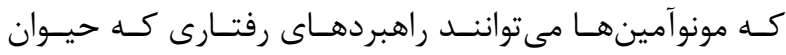

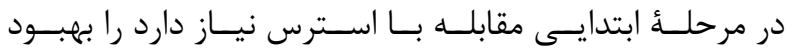

بخشـند.

\section{نورويِّتيدها}

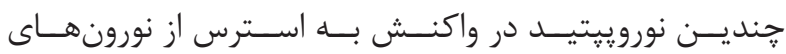

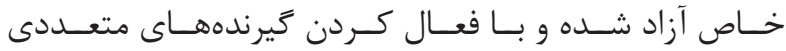

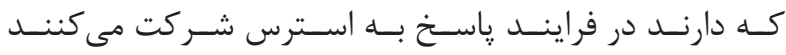

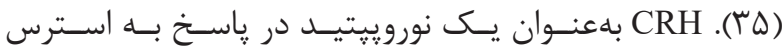

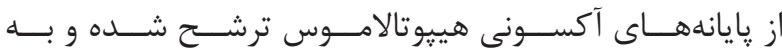

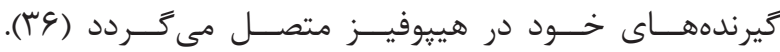

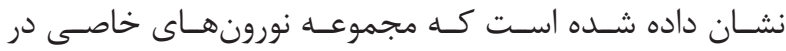

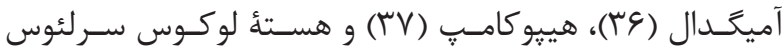

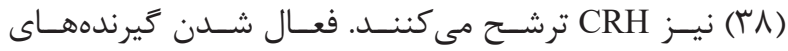

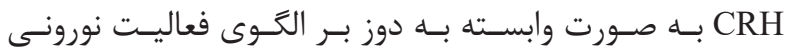

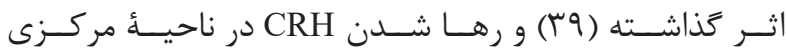

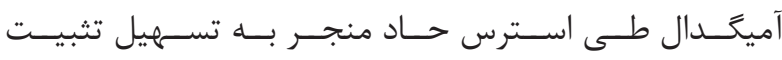

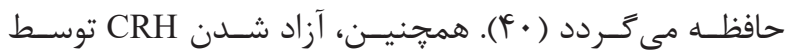

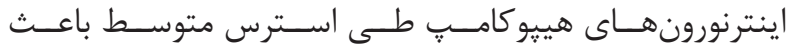

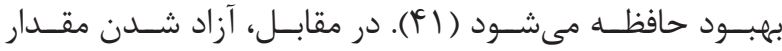

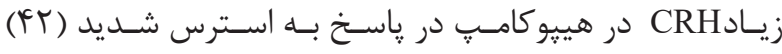

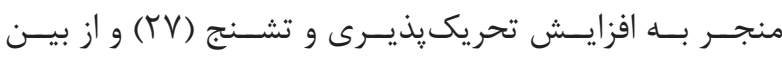

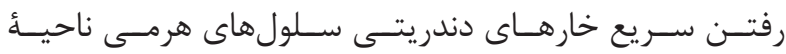
^CA3

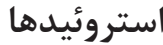

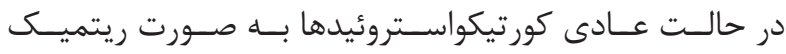

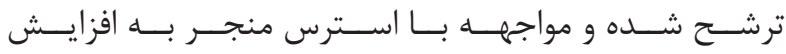

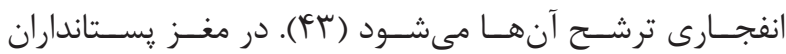

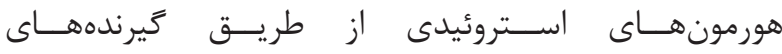

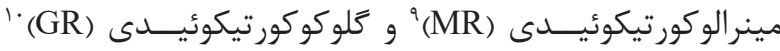

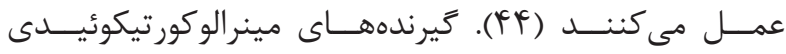

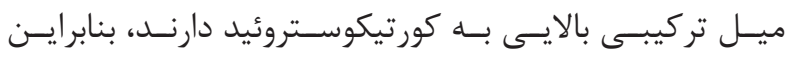

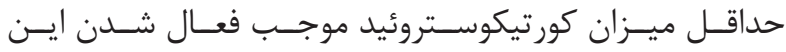

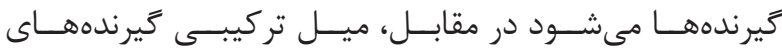

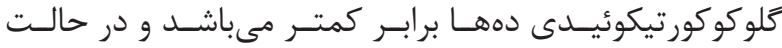

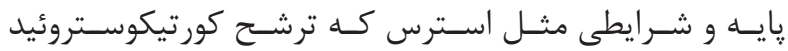

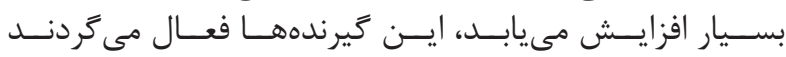

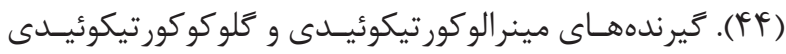

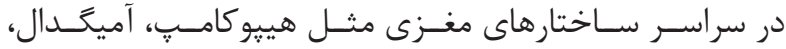

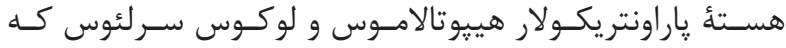

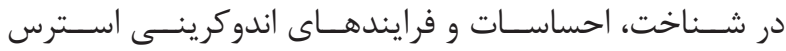

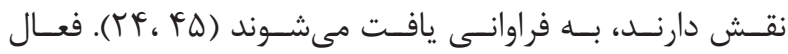

${ }^{7}$ Monoaminergic system ${ }^{8}$ Cornu ammonis area 3

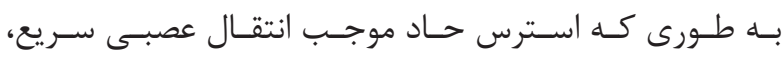

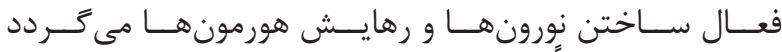

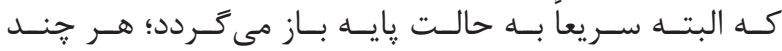

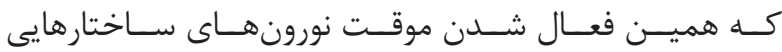

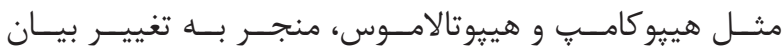

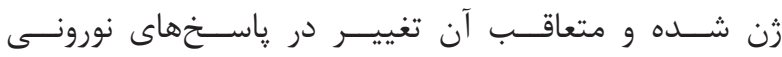

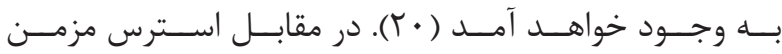

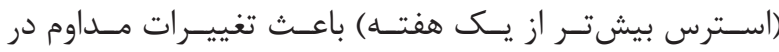

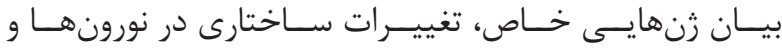

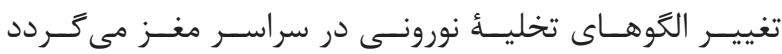

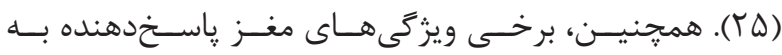

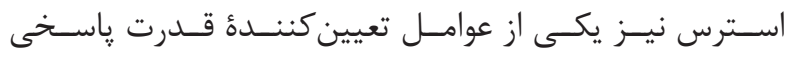

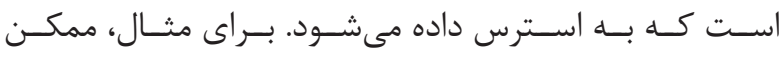

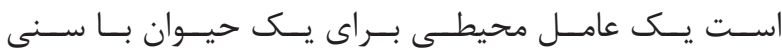

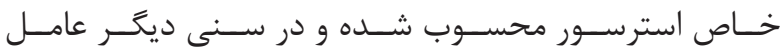

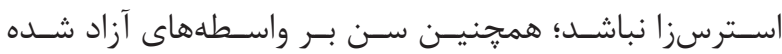

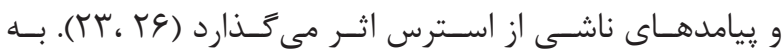

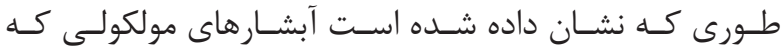

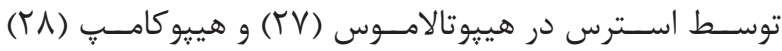

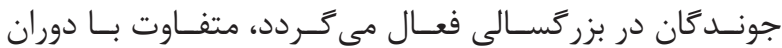

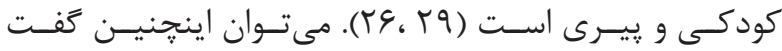

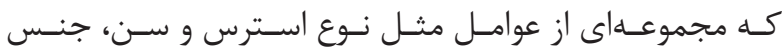

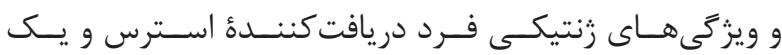

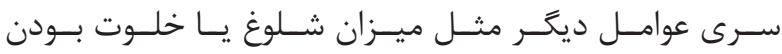

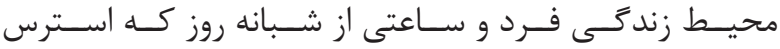

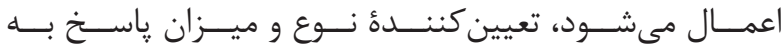

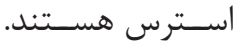

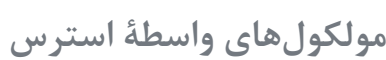
مونو آمين ها

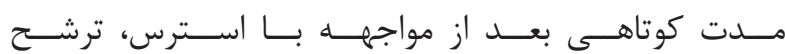

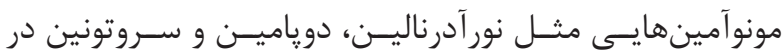

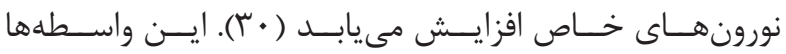

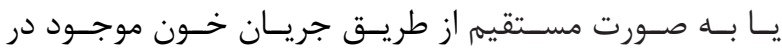

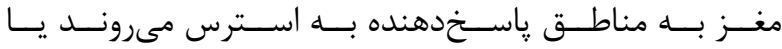

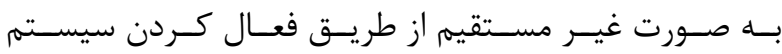

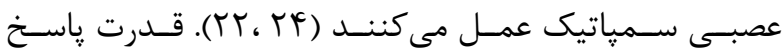

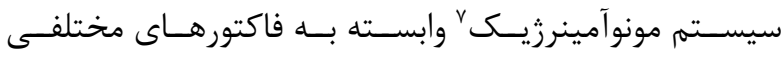

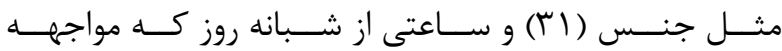

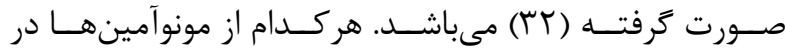

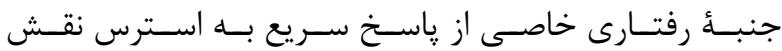

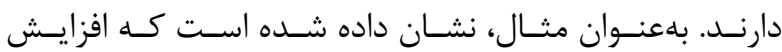

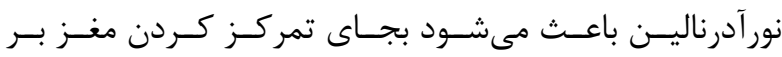

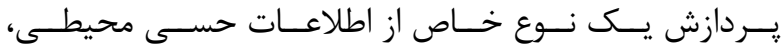

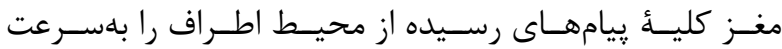

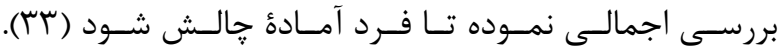

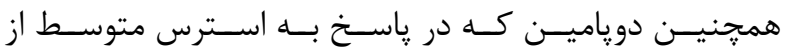

${ }^{9}$ Mineralocorticoid receptor

${ }^{10}$ Glucocorticoid receptor 


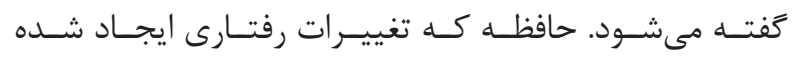

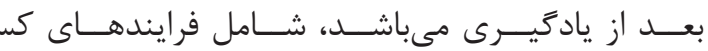

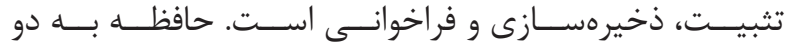

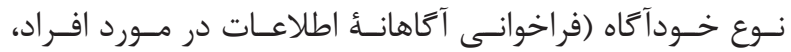

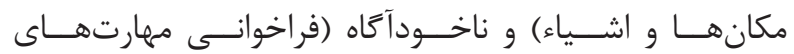

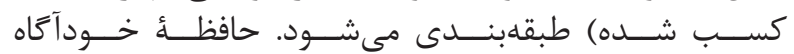

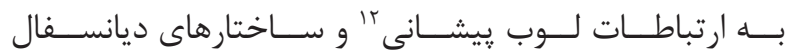

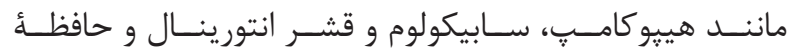

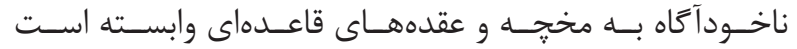

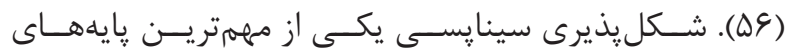

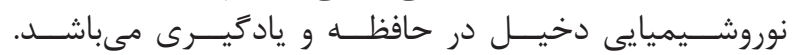

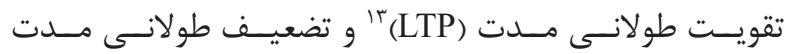
(ITD)

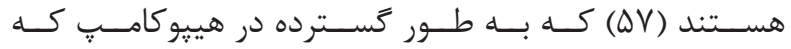

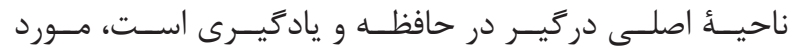

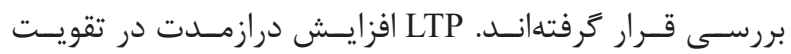

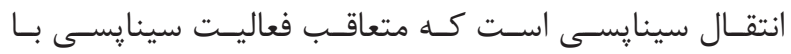

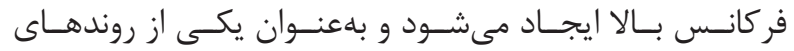

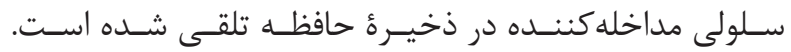

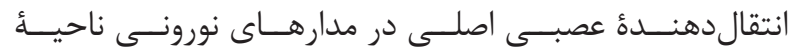

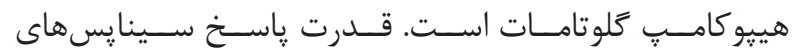

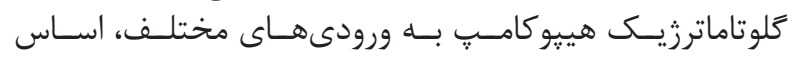

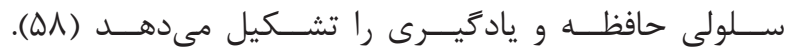

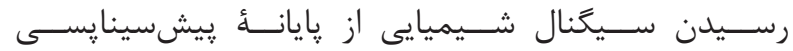

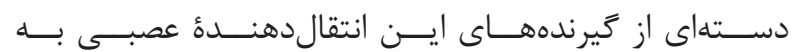

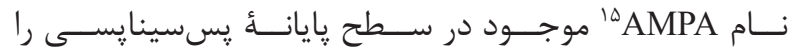

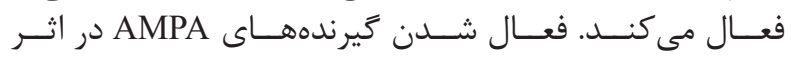

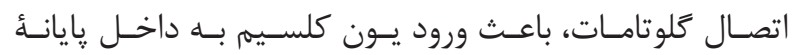

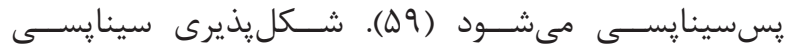

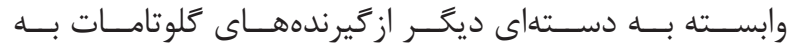

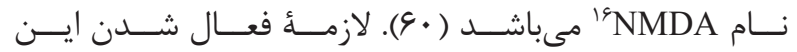

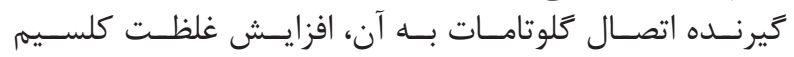

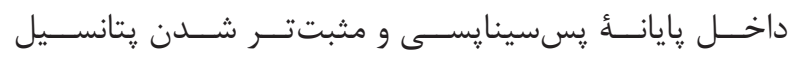

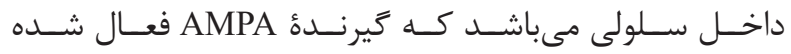

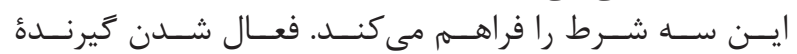

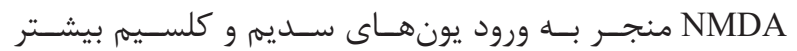

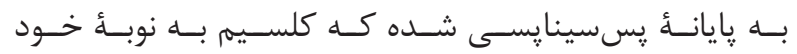

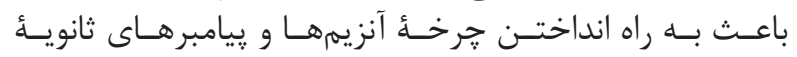

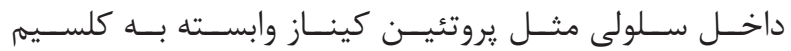

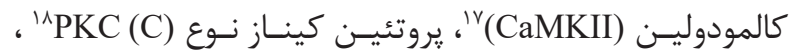

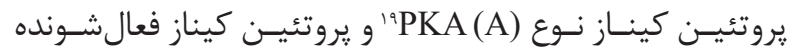

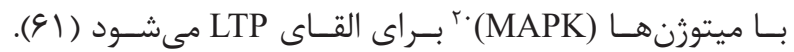

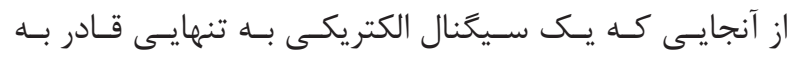

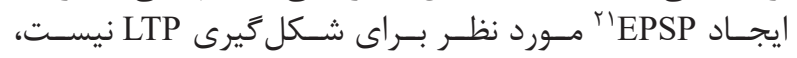

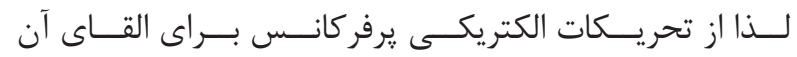

\footnotetext{
${ }^{11}$ Programming

${ }^{12}$ Frontal lobe

${ }^{13}$ Long-term potentiation

${ }^{14}$ Long-term depression

${ }^{15} \alpha$-amino-3-hydroxy-5-methyl-4-isoxazolepropionic acid

${ }^{16} \mathrm{~N}$-Methyl-D-aspartate
}

شـدن كيرندههـاى MR در هييوكامـٍ بــراى حفـظ اطلاعـات

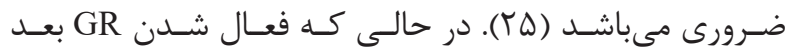

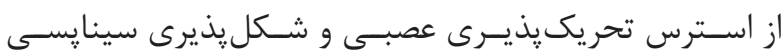

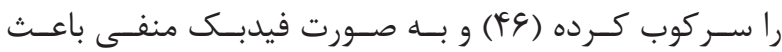

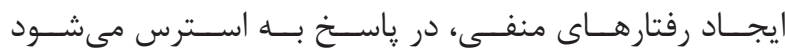

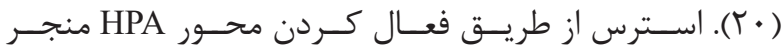

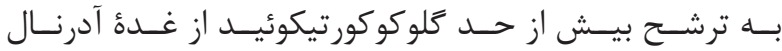

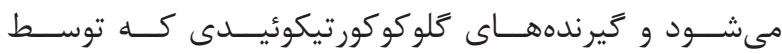

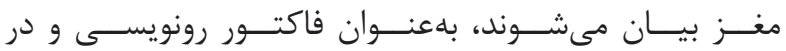

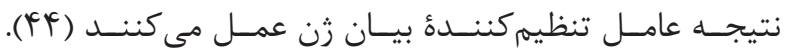

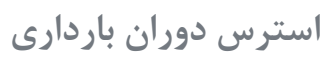

مواجهـهـ با اسـترس در ابتـداى زندگَى اثـر برنامهريزى كنـنده"

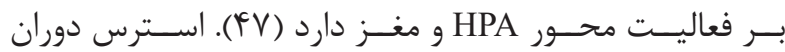

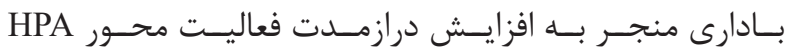

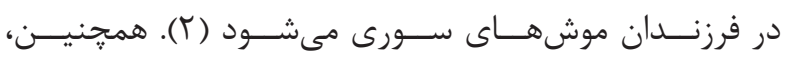

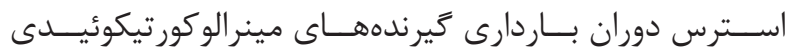

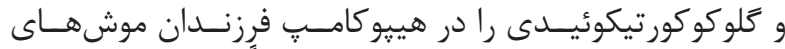

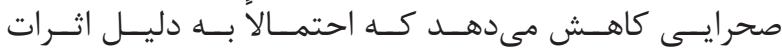

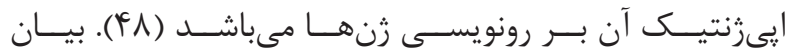

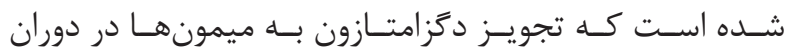

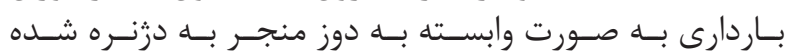

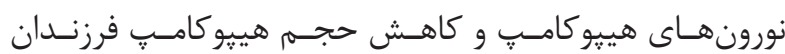

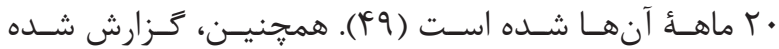

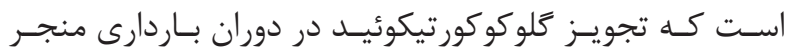

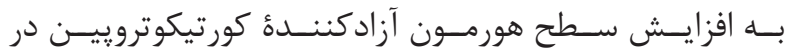

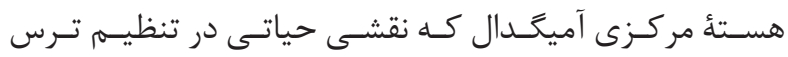

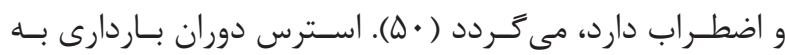

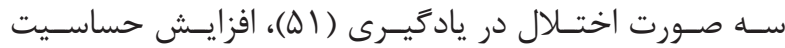

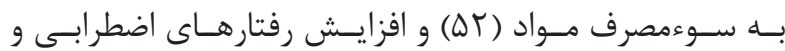
افسـرد

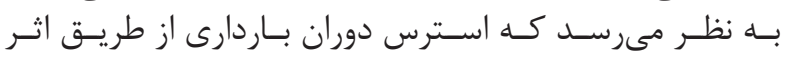

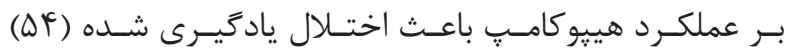

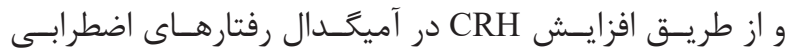

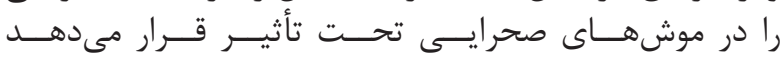

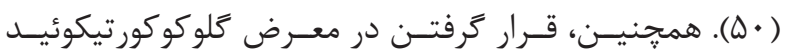

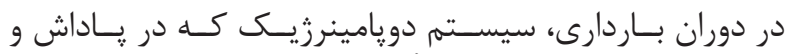

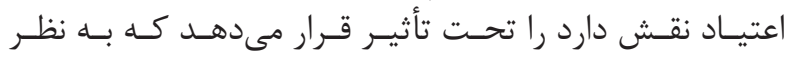

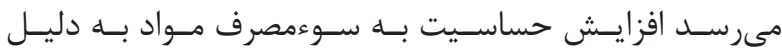

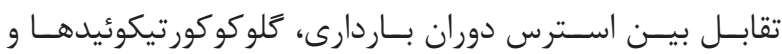

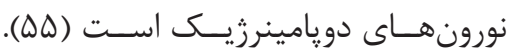

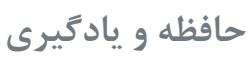

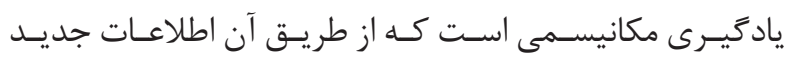

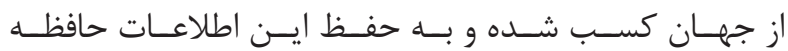

${ }^{17} \mathrm{Ca} 2+/$ calmodulin-dependent protein kinase II

${ }^{18}$ Protein kinase $\mathrm{C}$

${ }^{19}$ Protein kinase A

${ }^{20}$ Mitogen-activated protein kinase

${ }^{21}$ Excitatory postsynaptic potential 


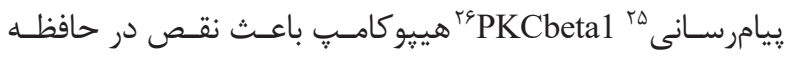

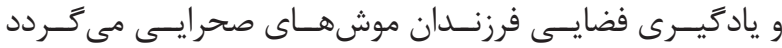

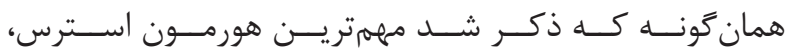

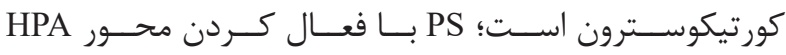

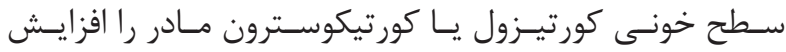

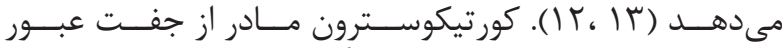

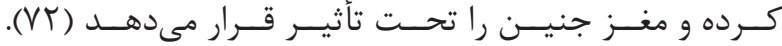

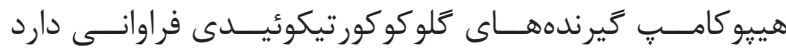

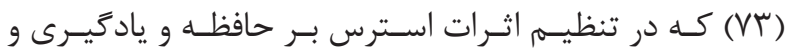

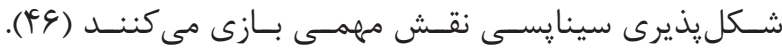

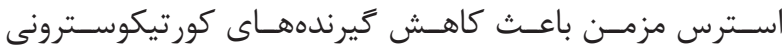

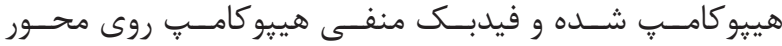

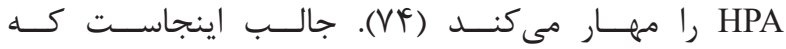

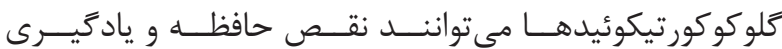

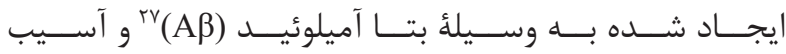

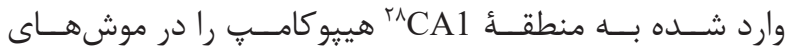
صحرايسى تشــديد كننــد (VD).

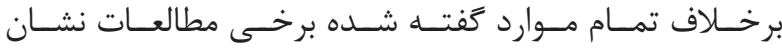

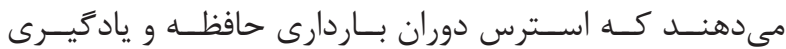

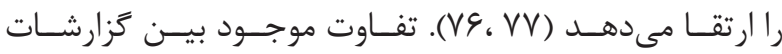

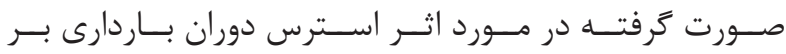

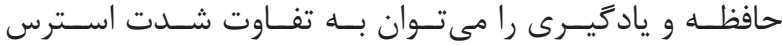

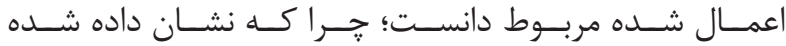

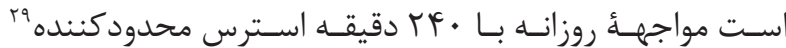

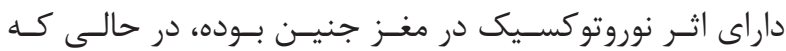

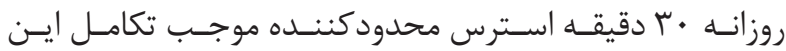

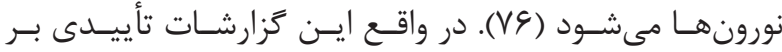

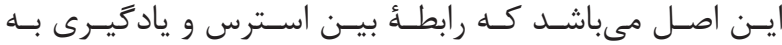

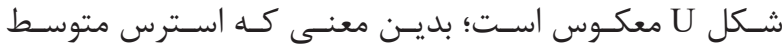

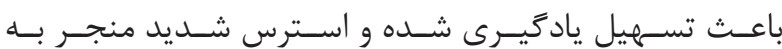

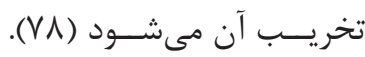

استرس دوران باردارى بر شكل يذيرى سينا پيسى

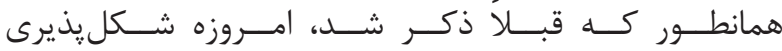

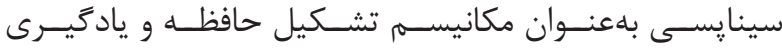

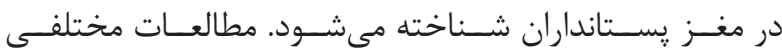

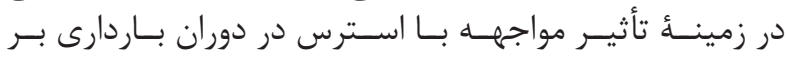

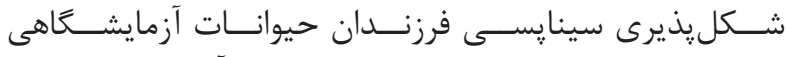

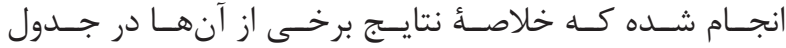

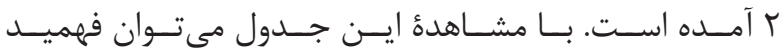

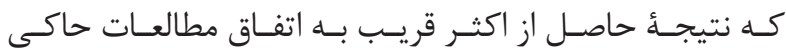

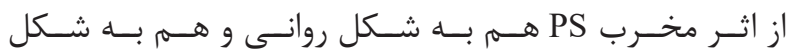

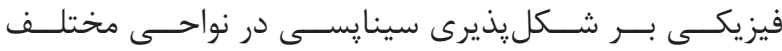

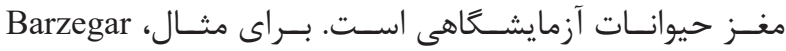

${ }^{22}$ Neurogenesis

${ }^{23}$ Cornu ammonis dentate gyrus

${ }^{24}$ cAMP response element-binding protein mRNA

${ }^{25}$ Signaling
اســتفاده مى گَــردد (1).

اثرات استرس دوران باردارى بر حافظه و يادَيرى مطالعـات مختلـف انجــام شــده روى انسـان و مدل هــاى حيوانى

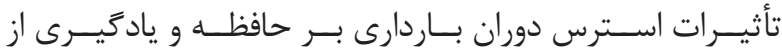

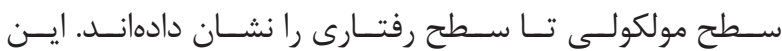

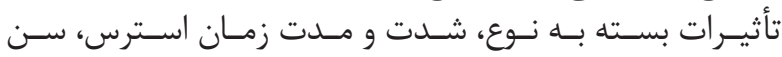

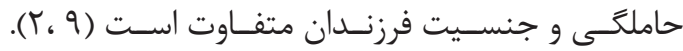

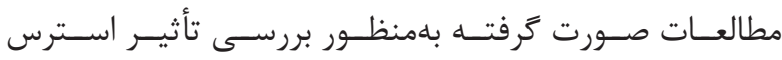

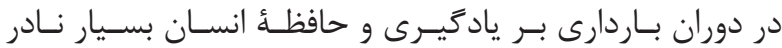

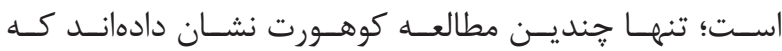

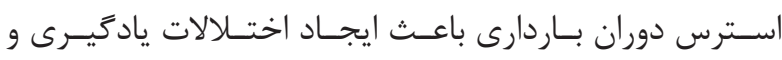

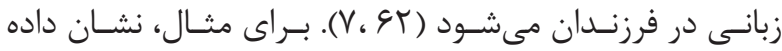

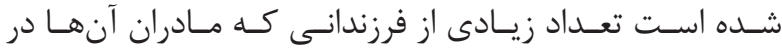

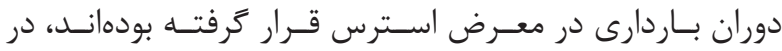

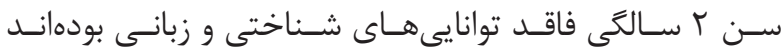

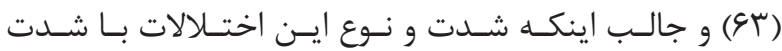

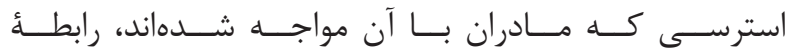

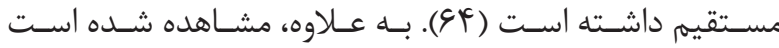

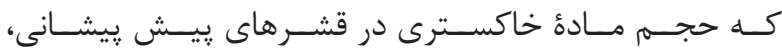

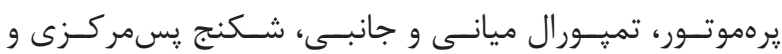

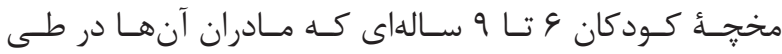

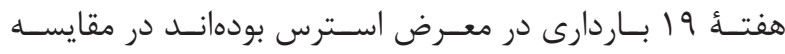

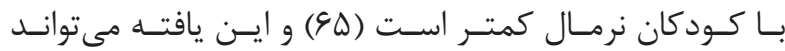

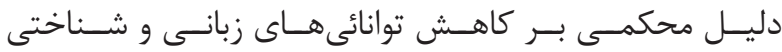

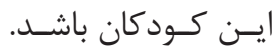

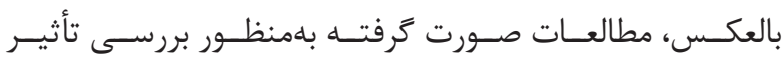

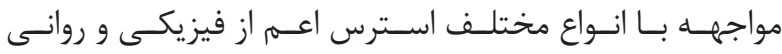

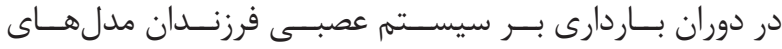

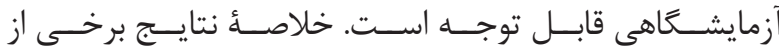

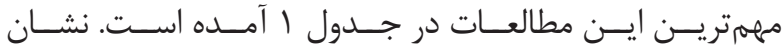

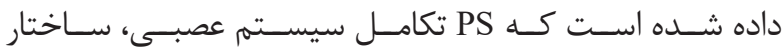

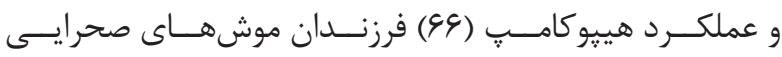

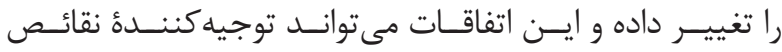

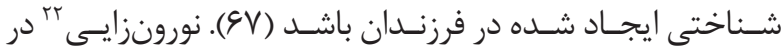

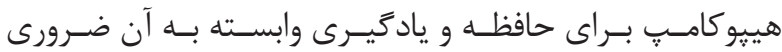

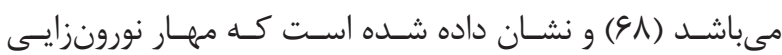

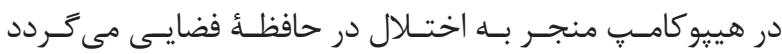

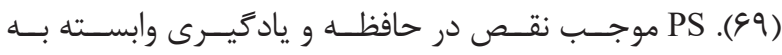

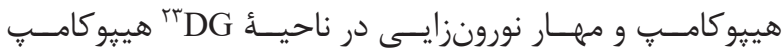

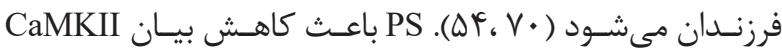

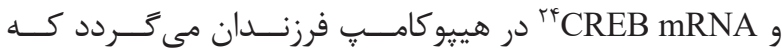

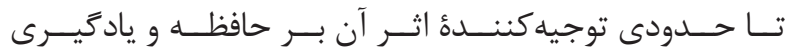

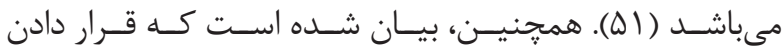

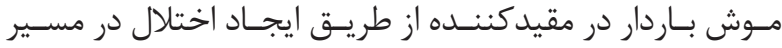

\footnotetext{
${ }^{26}$ Protein kinase $\mathrm{C}$ betal

${ }^{27}$ Amyloid beta

${ }^{28}$ Cornu Ammonis area 1

${ }^{29}$ Restraint stress
} 


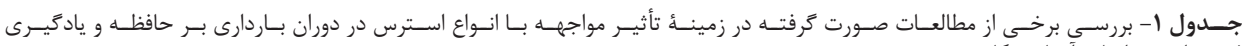

\begin{tabular}{|c|c|c|c|c|c|}
\hline اتو ير خالطاله و يادئيرى & كرزئنان & 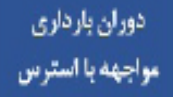 & لوع السترس & توئه & عنبع \\
\hline نغريب ياكيرى نضائ & 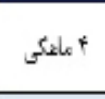 & S: & 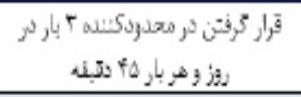 & 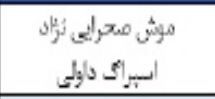 & (Qf) \\
\hline 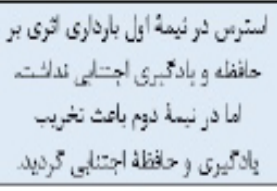 & ज5 & 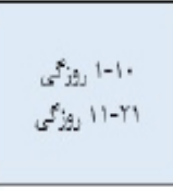 & 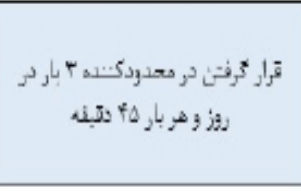 & 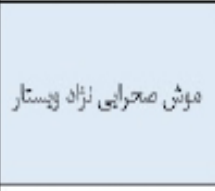 & (V9) \\
\hline تغريب ياكُمبرى لضايى & Stog & $S^{5}|A| 0-Y \mid$, & روزله ا سايد استرب حوتى & موش عدرائي تئزاد & (A.) \\
\hline 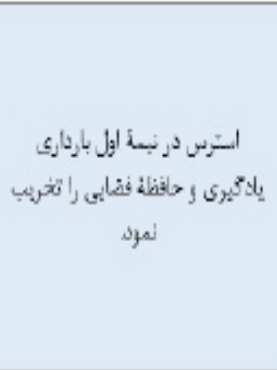 & آ عاعكى & 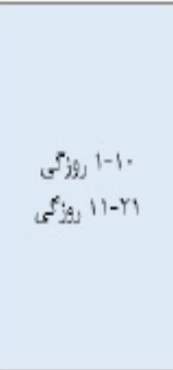 & 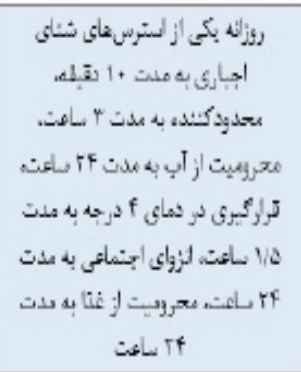 & عرش صترائ نزلاد يوبتار & (Al) \\
\hline 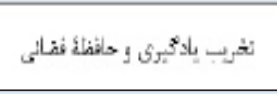 & 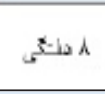 & 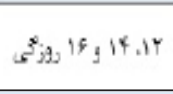 & 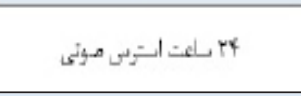 & 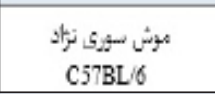 & $(\Psi)$ \\
\hline 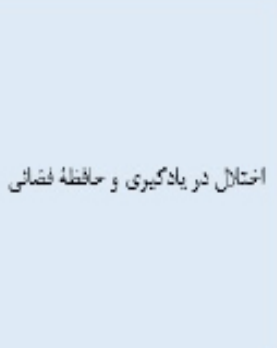 & 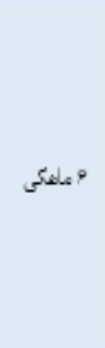 & r. & 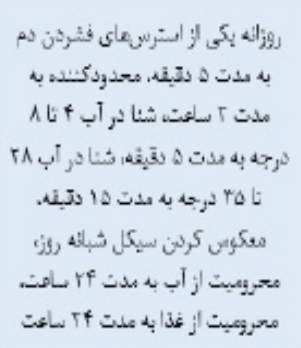 & $\begin{array}{c}\text { عوش سوري نزلا } \\
\text { APPawe/PS1dE9 }\end{array}$ & (ब) \\
\hline تخريب بادكيرى و حالقطأ لضاتى & sor or & 促 & 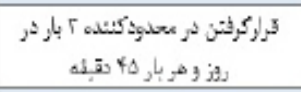 & موثن سورى نراد CF-1 & $(v \cdot)$ \\
\hline 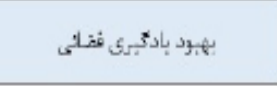 & 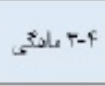 & 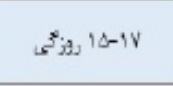 & 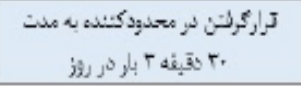 & موش صترايع نزياد & $\left.(v)^{\prime}\right)$ \\
\hline 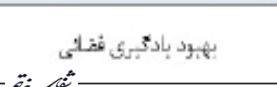 & $\begin{array}{l}r k-F r \\
\text { Sigs }\end{array}$ & 仿 & 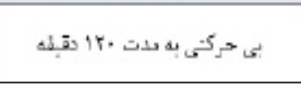 & 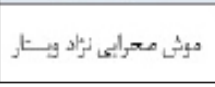 & $(m)$ \\
\hline
\end{tabular}

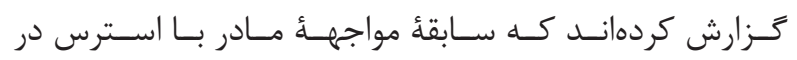

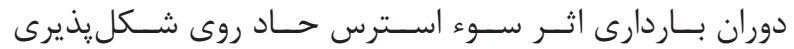

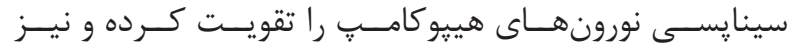

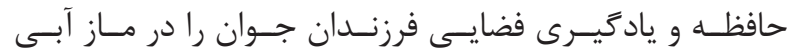

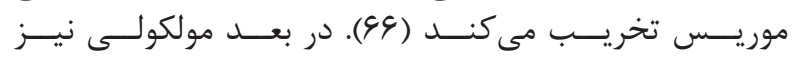

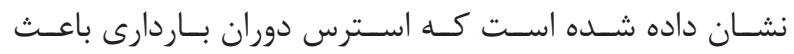

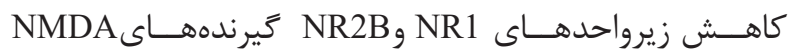

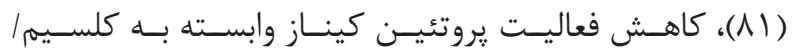

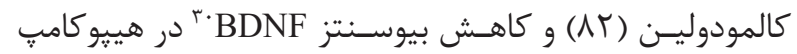

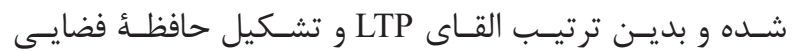

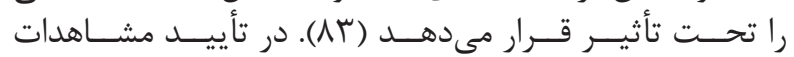

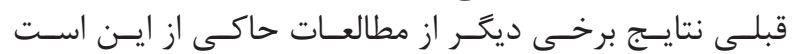

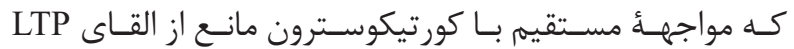

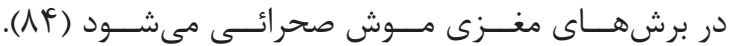

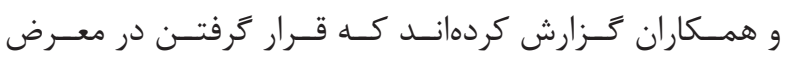

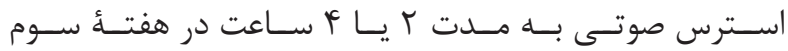

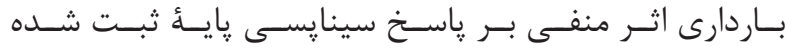

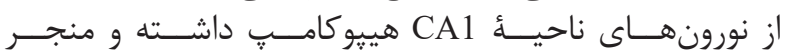

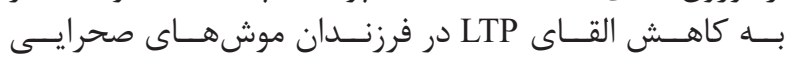

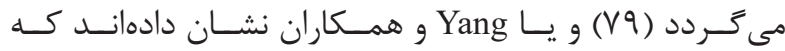

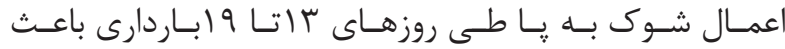

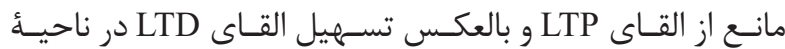

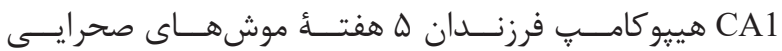

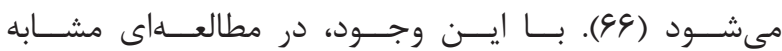

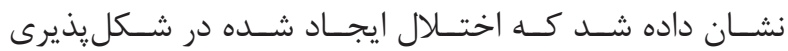

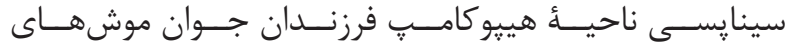

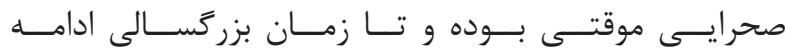

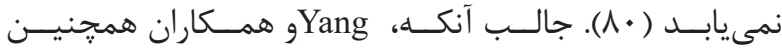




\begin{tabular}{|c|c|c|c|c|c|}
\hline التر يو ثكل بذيزي سيثابسى & سئ فرزندان & 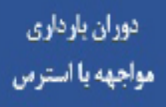 & 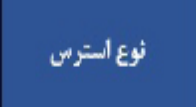 & " & لمنيع \\
\hline 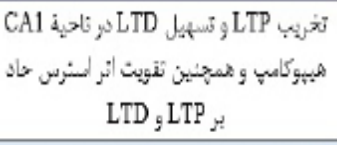 & 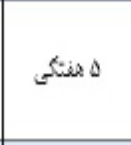 & S.jog 19 & 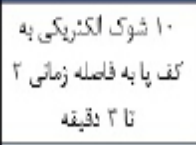 & مؤش صحرائى نزلة ويسئار & (94) \\
\hline 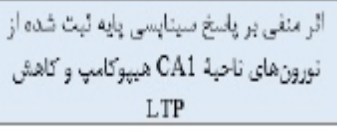 & Y & $5 j g, 10-r 1$ & 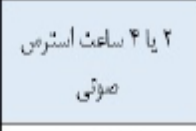 & 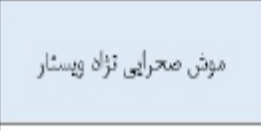 & (Ar) \\
\hline 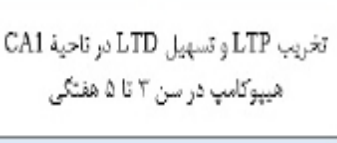 & ا & Sigs $1 a-r \mid$ & 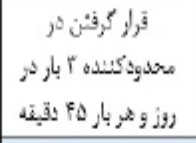 & عبر صبرابي نزاء لسيراث ناولى & (AT) \\
\hline كخريب LTP در نامبل CA1 غيبركالي & ه & Sig $|Y-T|$ & 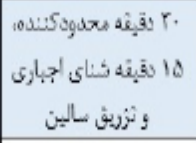 & 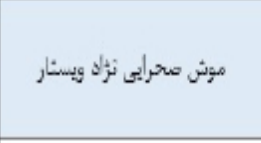 & $(A)$ \\
\hline كخريب LTP در نامبة CA1 غييوكامب & ס ס קوز5 & S & 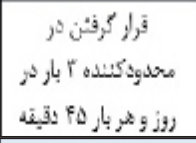 & مؤش صترائ نثلاث ويسثار & (A9) \\
\hline تخريب LTP درقير يشانى & Solor & (19-1) & 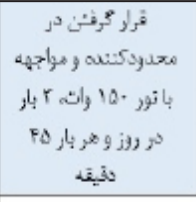 & 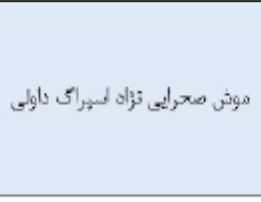 & (१.) \\
\hline 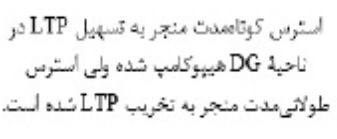 & ا هiت: & ل & 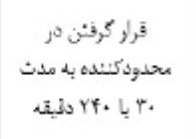 & موثش صترايب نزاه لسراثى داؤلى & (91) \\
\hline 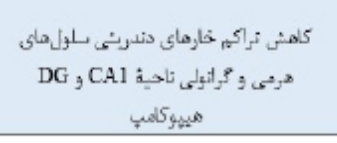 & D to tوز5 & Sig $1 \Upsilon 19$ & 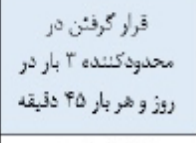 & موش صبرايع نزاد لبرائ داولى & (भi) \\
\hline 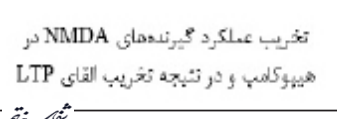 & STiex Y & 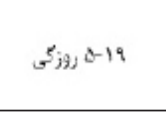 & 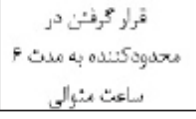 & ICR بوثن سورى تزوان & (hip) \\
\hline
\end{tabular}

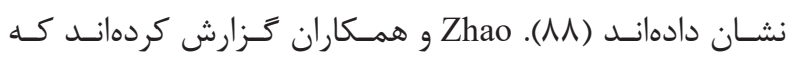

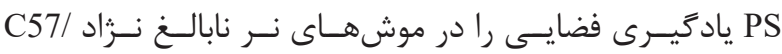

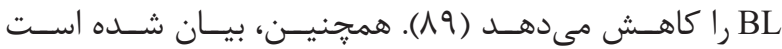

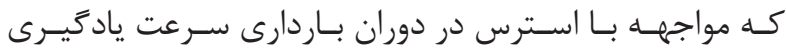

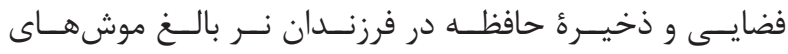

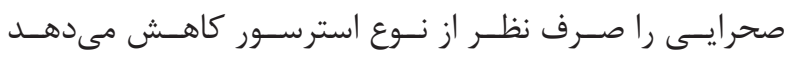

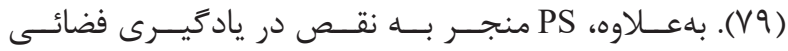

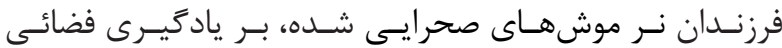

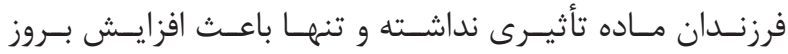

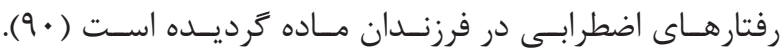

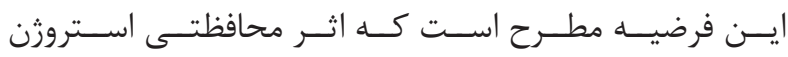

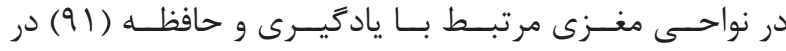

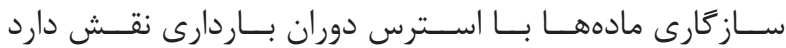

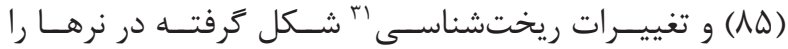

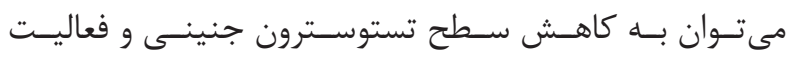

اثرات وابسته به جنسيت

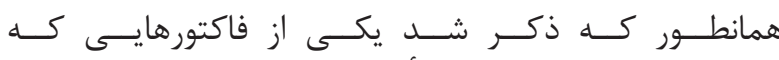

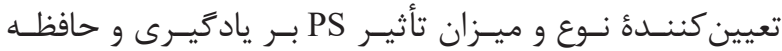

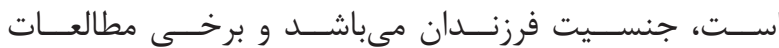

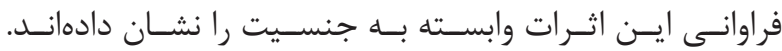

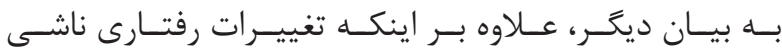

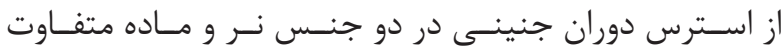

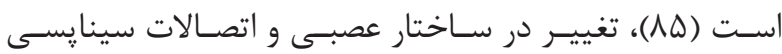

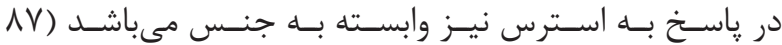

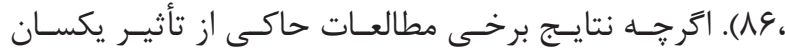

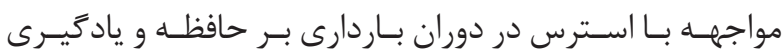

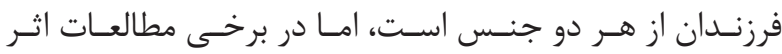

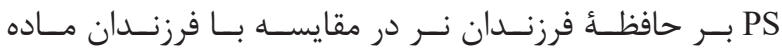

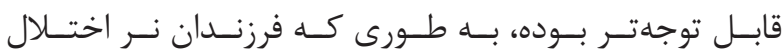

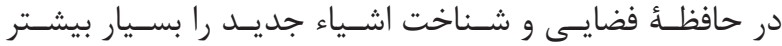




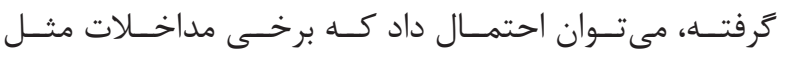

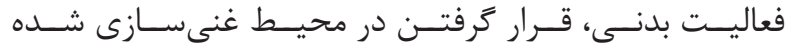

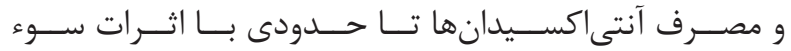

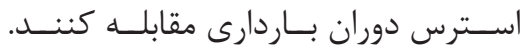

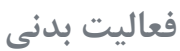

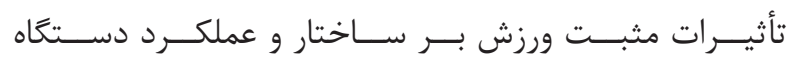

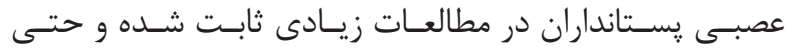

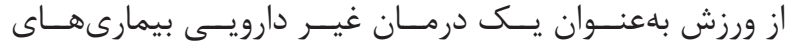

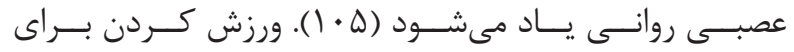

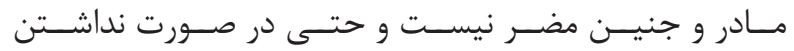

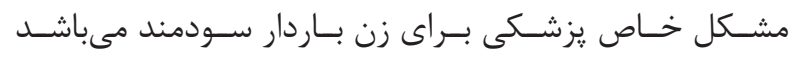

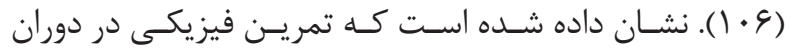

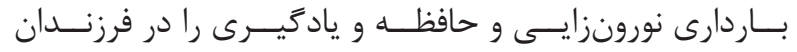

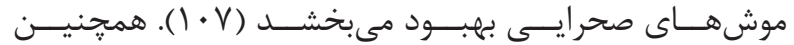

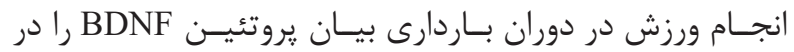

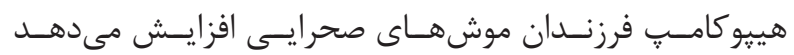

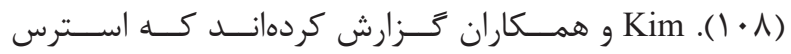

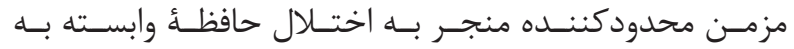

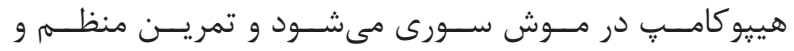

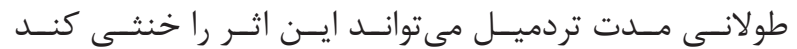

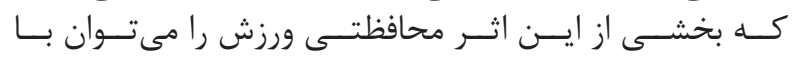

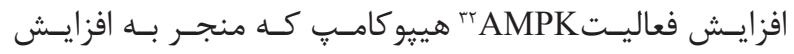

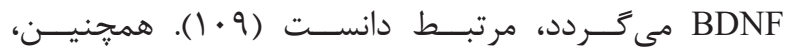

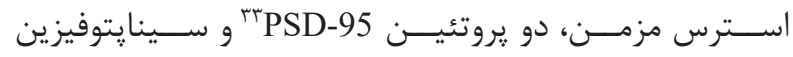

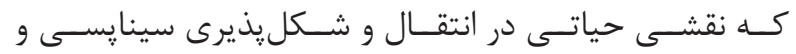

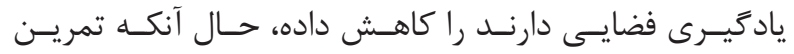

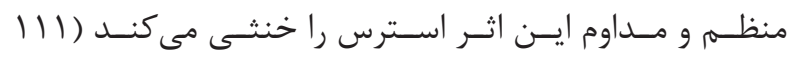

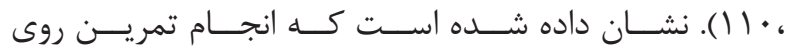

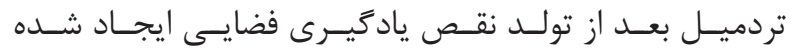

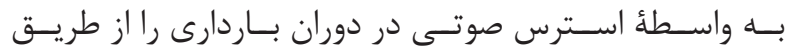

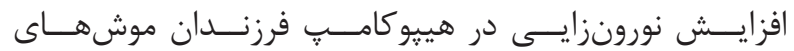

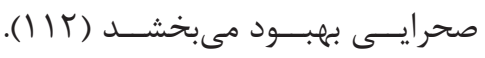
غنىسازى محيط

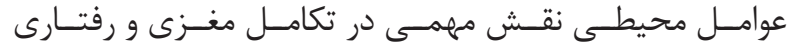

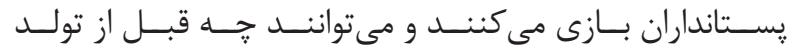

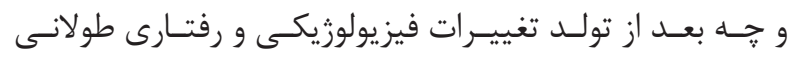

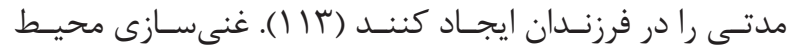

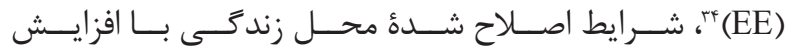

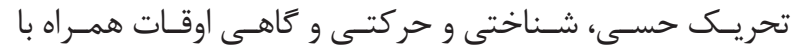

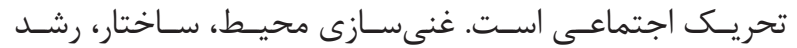

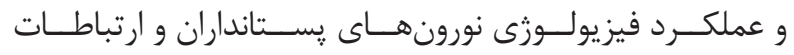

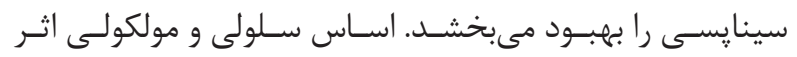

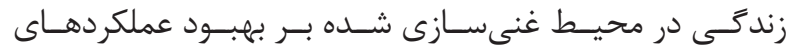

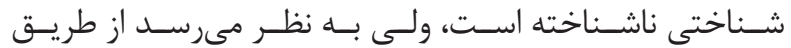

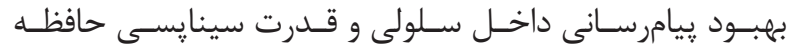

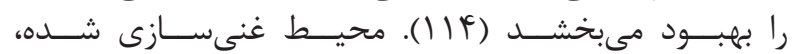

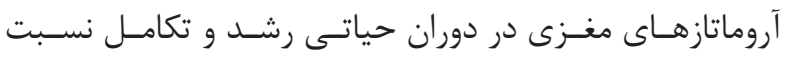

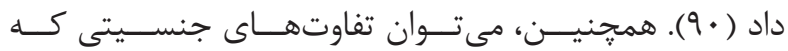

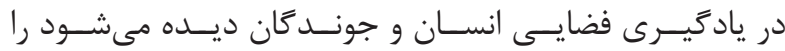

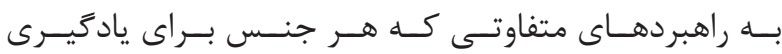

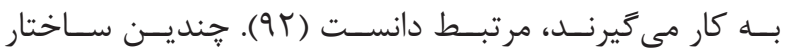

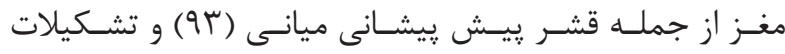

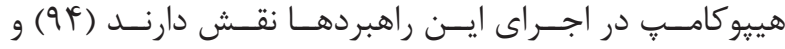

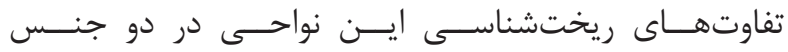

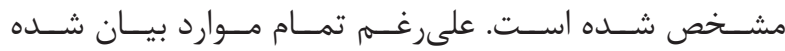

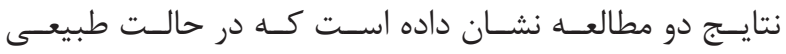

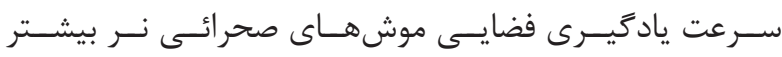

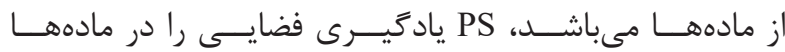

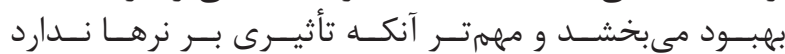

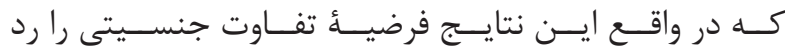

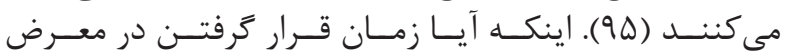

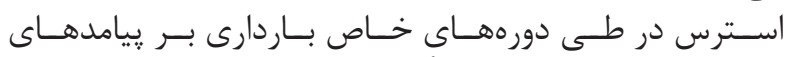

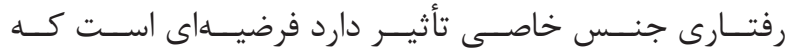

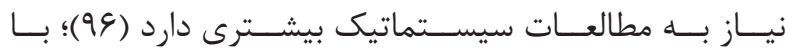

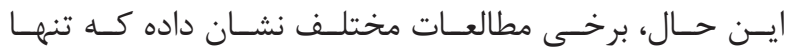

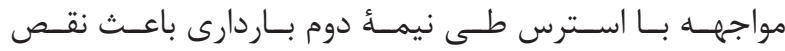

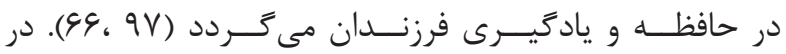

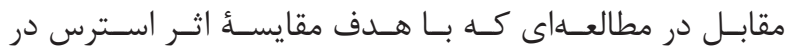

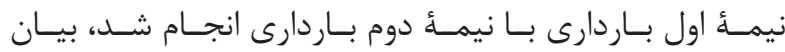

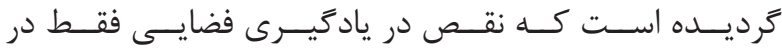

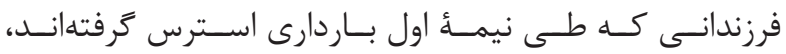

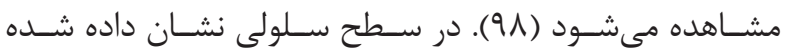

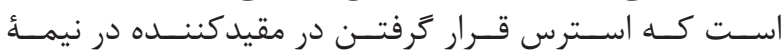

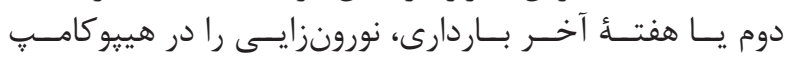

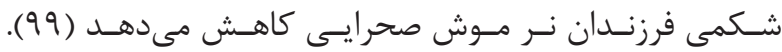

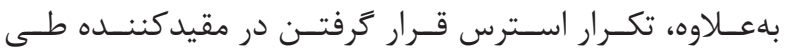

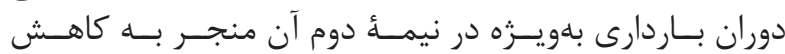

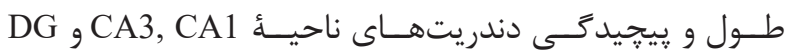

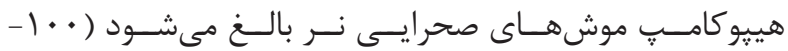

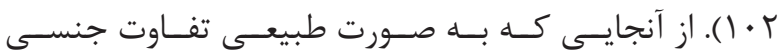

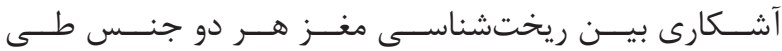

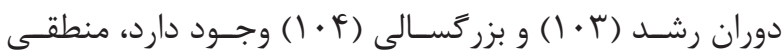

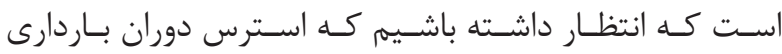

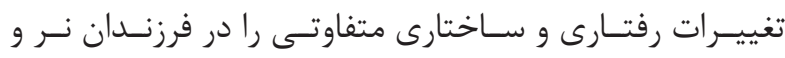

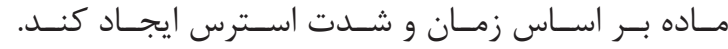

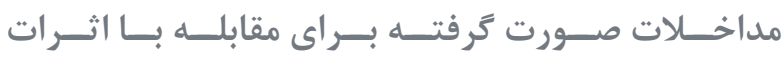
ســـوء اســـترس دوران بــاردارى

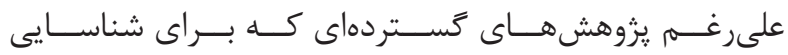

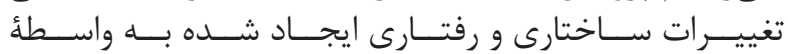

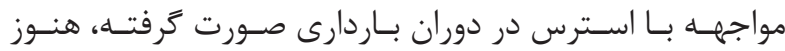

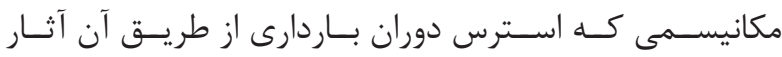

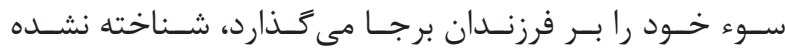

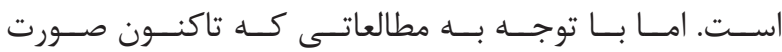




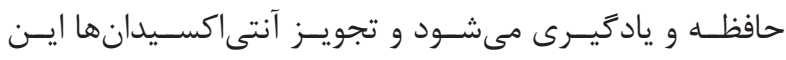

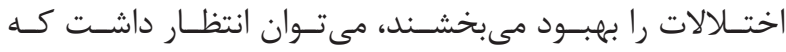

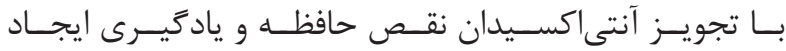

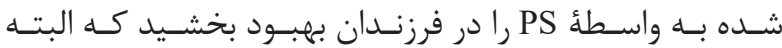

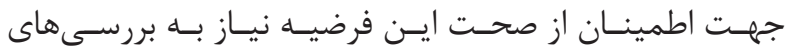

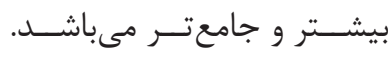
نتيجه تيرى

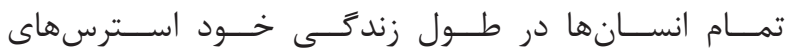

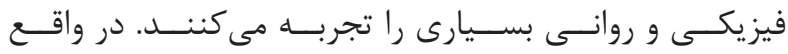

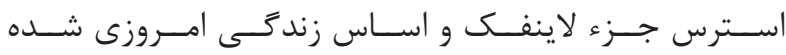

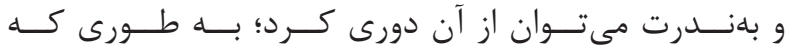

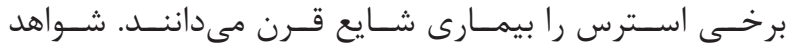

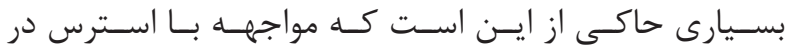

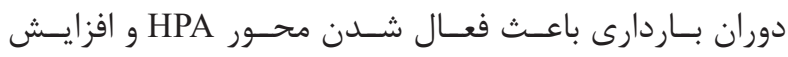

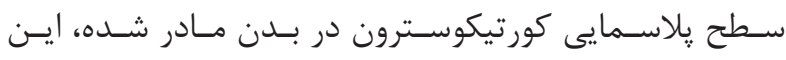

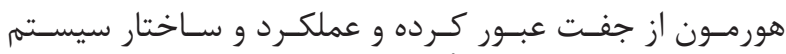

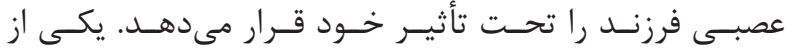

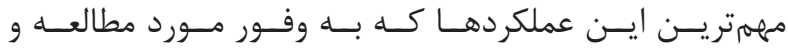

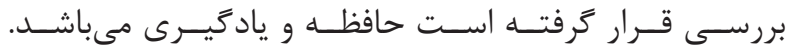

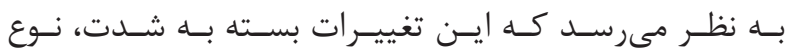

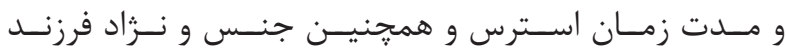

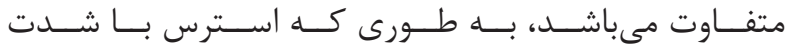

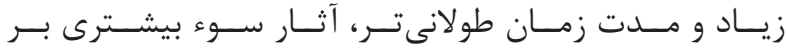

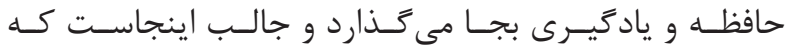

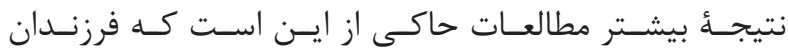

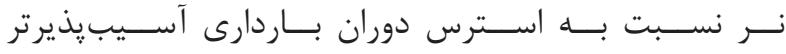

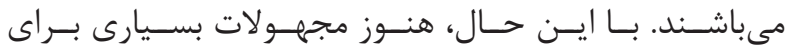

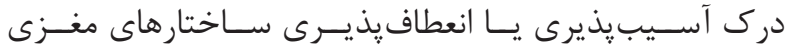

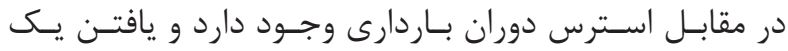

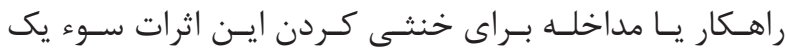

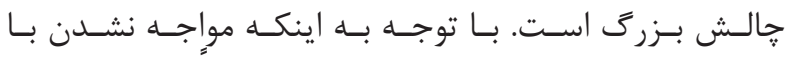

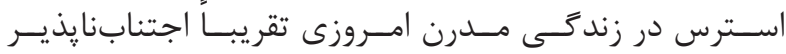

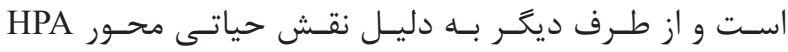

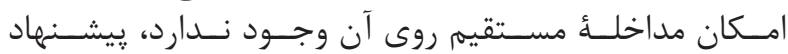

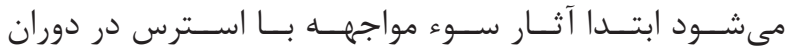

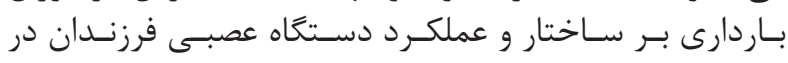

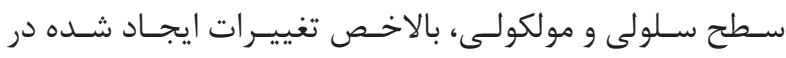

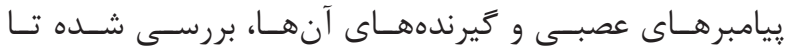

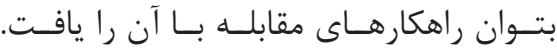

$$
\text { تشكر و قدردانى }
$$

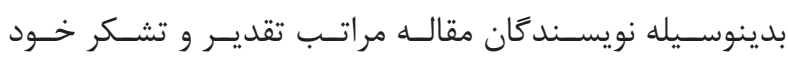

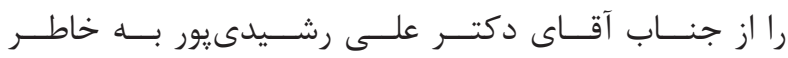

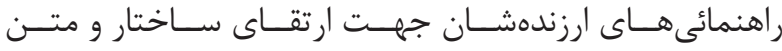

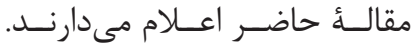

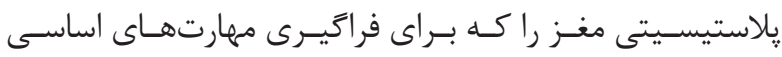

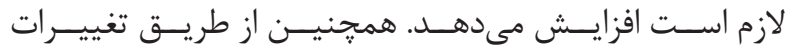

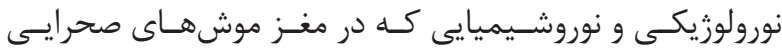

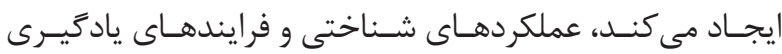

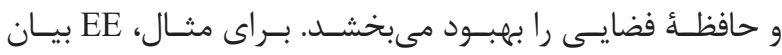

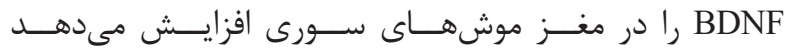

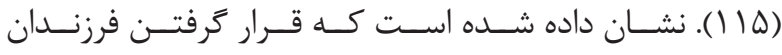

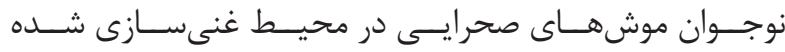

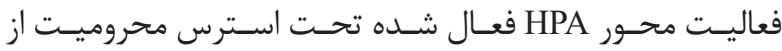

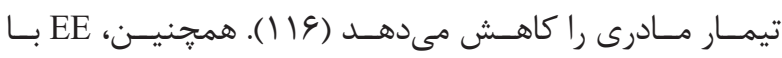

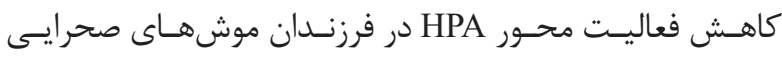

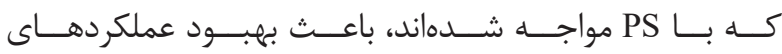

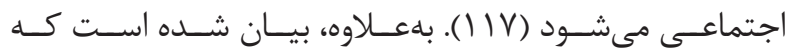

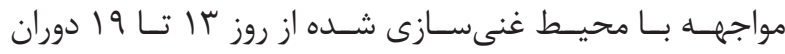

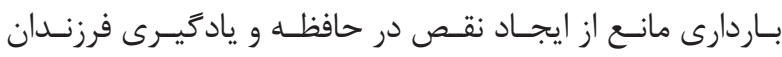

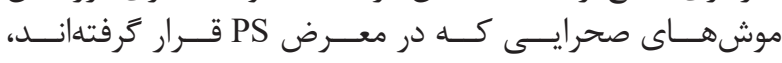

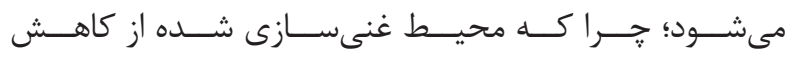

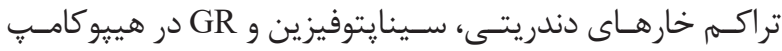

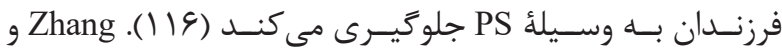

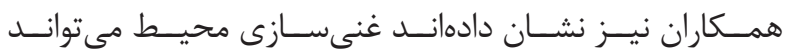

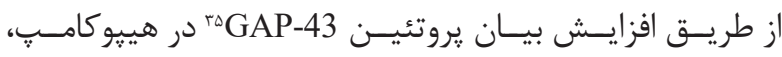

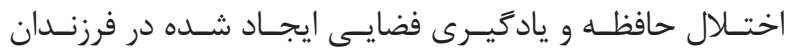

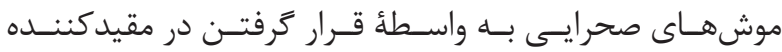

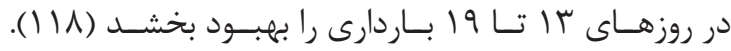

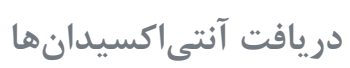

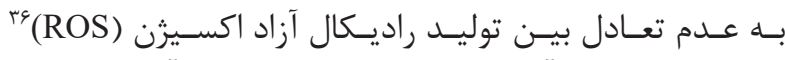

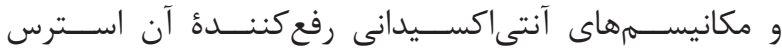

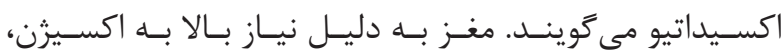

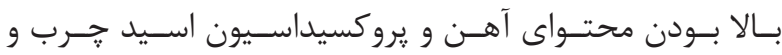

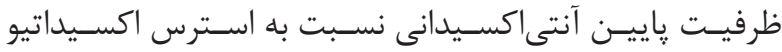

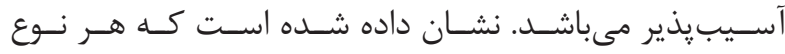

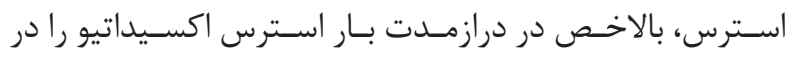

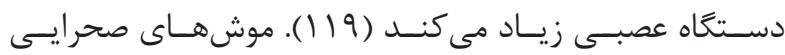

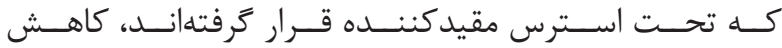

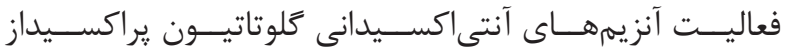

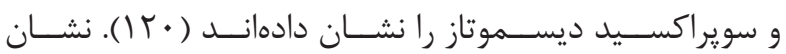

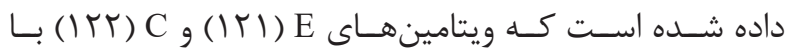

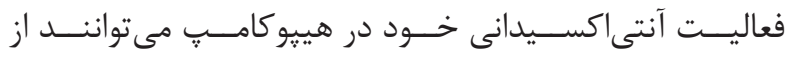

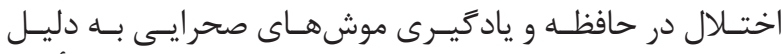

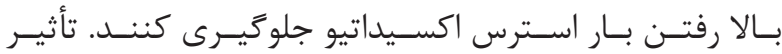

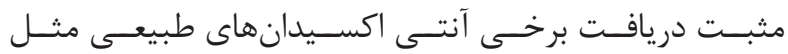

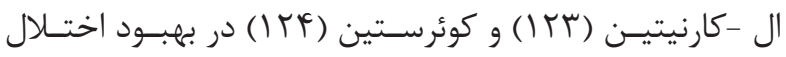

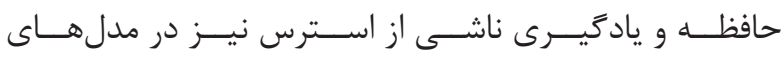

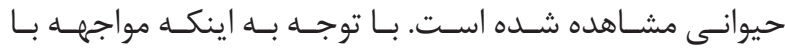

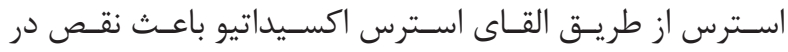


1. McEwen BS. Central effects of stress hormones in health and disease: Understanding the protective and damaging effects of stress and stress mediators. Eur J Pharmacol. 2008; 583(2-3): 174-85.

2. Jafari Z, Mehla J, Kolb BE, Mohajerani MH. Prenatal noise stress impairs HPA axis and cognitive performance in mice. Sci Rep. 2017; 7(1): 10560. doi: 10.1038/ s41598-017-09799-6.

3. Matthews SG. Antenatal glucocorticoids and programming of the developing CNS. Pediatr Res. 2000; 47(3): 291-300.

4. Tang W, Cheng J, Wang Z-Y, Chen K-Y, Han Z-M, Wang Q-H, et al. The synergistic roles of the chronic prenatal and offspring stress exposures in impairing offspring learning and memory. J Alzheimers Dis. 2016; 53(1): 221-36.

5. Myers RE. Maternal psychological stress and fetal asphyxia: a study in the monkey. Am J Obstet Gynecol. 1975; 122(1): 47-59.

6. Cao X, Laplante DP, Brunet A, Ciampi A, King S. Prenatal maternal stress affects motor function in $5 \frac{1}{2-}$ year-old children: Project Ice Storm. Dev Psychobiol. 2014; 56(1): 117-25.

7. King S, Laplante DP. The effects of prenatal maternal stress on children's cognitive development: Project Ice Storm. Stress. 2005; 8(1): 35-45.

8. Patchev VK, Patchev AV. Experimental models of stress. Dialogues Clin Neurosci. 2006; 8(4): 417-32.

9. Ellman LM, Schetter CD, Hobel CJ, Chicz-DeMet A, Glynn LM, Sandman CA. Timing of fetal exposure to stress hormones: effects on newborn physical and neuromuscular maturation. Dev Psychobiol. 2008; 50(3): 232-41.

10. Neeley EW, Berger R, Koenig JI, Leonard S. Strain dependent effects of prenatal stress on gene expression in the rat hippocampus. Physiol Behav. 2011; 104(2): 334-9.

11. Graignic-Philippe R, Dayan J, Chokron S, Jacquet A, Tordjman S. Effects of prenatal stress on fetal and child development: a critical literature review. Neurosci Biobehav Rev. 2014; 43: 137-62.

12. Ward HE, Johnson EA, Salm AK, Birkle DL. Effects of prenatal stress on defensive withdrawal behavior and corticotropin releasing factor systems in rat brain. Physiol Behav. 2000; 70(3-4): 359-66.
13. Szuran TF, Pliška V, Pokorny J, Welzl H. Prenatal stress in rats: effects on plasma corticosterone, hippocampal glucocorticoid receptors, and maze performance. Physiol Behav. 2000; 71(3-4): 353-62.

14. Gitau R, Fisk NM, Teixeira JM, Cameron A, Glover V. Fetal hypothalamic-pituitary-adrenal stress responses to invasive procedures are independent of maternal responses 1. T J Clin Endocrinol Metab. 2001; 86(1): 104-9.

15. Howerton CL, Bale TL. Prenatal programing: at the intersection of maternal stress and immune activation. Horm Behav. 2012; 62(3): 237-42.

16. Weinstock M. The long-term behavioural consequences of prenatal stress. Neurosci Biobehav Rev. 2008; 32(6): 1073-86.

17. Van den Bergh BR, Mulder EJ, Mennes M, Glover V. Antenatal maternal anxiety and stress and the neurobehavioural development of the fetus and child: links and possible mechanisms. Neurosci Biobehav Rev. 2005; 29(2): 237-58.

18. Madalena KM, Lerch JK. The effect of glucocorticoid and glucocorticoid receptor interactions on brain, spinal cord, and glial cell plasticity. Neural Plasticity. 2017; 2017.

19. Harris A, Seckl J. Glucocorticoids, prenatal stress and the programming of disease. Horm Behav. 2011; 59(3): 279-89.

20. Joëls M, Baram TZ. The neuro-symphony of stress. Nat Rev Neurosci. 2009; 10(6): 459-66.

21. McEwen BS. Physiology and neurobiology of stress and adaptation: central role of the brain. Physiol Rev. 2007; 87(3): 873-904.

22. Ulrich-Lai YM, Herman JP. Neural regulation of endocrine and autonomic stress responses. Nat Rev Neurosci. 2009; 10(6): 397-409.

23. Fenoglio KA, Brunson KL, Baram TZ. Hippocampal neuroplasticity induced by early-life stress: functional and molecular aspects. Front Neuroendocrinol. 2006; 27(2): 180-92.

24. McGaugh JL. The amygdala modulates the consolidation of memories of emotionally arousing experiences. Annu Rev Neurosci. 2004; 27: 1-28.

25. Joëls M, Karst H, Krugers HJ, Lucassen PJ. Chronic stress: implications for neuronal morphology, function 
and neurogenesis. Front Neuroendocrinol. 2007; 28(23): 72-96.

26. Lupien SJ, Fiocco A, Wan N, Maheu F, Lord C, Schramek T, et al. Stress hormones and human memory function across the lifespan. Psychoneuroendocrinology. 2005; 30(3): 225-42.

27. Baram TZ, Hatalski CG. Neuropeptide-mediated excitability: a key triggering mechanism for seizure generation in the developing brain. Trends Neurosci. 1998; 21(11): 471-6.

28. Chen Y, Fenoglio K, Dube C, Grigoriadis D, Baram T. Cellular and molecular mechanisms of hippocampal activation by acute stress are age-dependent. Mol Psychiatry. 2006; 11(11): 992-1002.

29. Brunson KL, Krami`r E, Lin B, Chen Y, Colgin LL, Yanagihara TK, et al. Mechanisms of late-onset cognitive decline after early-life stress. J Neurosci. 2005; 25(41): 9328-38.

30. Maier SF, Watkins LR. Stressor controllability and learned helplessness: the roles of the dorsal raphe nucleus, serotonin, and corticotropin-releasing factor Neurosci Biobehav Rev. 2005; 29(4-5): 829-41.

31. Mitsushima D, Yamada K, Takase K, Funabashi T, Kimura F. Sex differences in the basolateral amygdala: the extracellular levels of serotonin and dopamine, and their responses to restraint stress in rats. Eur J Neurosci. 2006; 24(11): 3245-54.

32. Piazza PV, Rougé-Pont F, Deroche V, Maccari S, Simon H, Le Moal M. Glucocorticoids have statedependent stimulant effects on the mesencephalic dopaminergic transmission. Proc Natl Acad Sci U S A. 1996; 93(16): 8716-20.

33. Aston-Jones G, Cohen JD. An integrative theory of locus coeruleus-norepinephrine function: adaptive gain and optimal performance. Annu Rev Neurosci. 2005; 28: $403-50$.

34. Park H-J, Shim HS, Lee S, Hahm DH, Lee H, Oh $\mathrm{CT}$, et al. Anti-stress effects of human placenta extract: possible involvement of the oxidative stress system in rats. BMC Complement Altern Med. 2018; 18(1): 149. doi: 10.1186/s12906-018-2193-x.

35. Mathew SJ, Price RB, Charney DS. Recent advances in the neurobiology of anxiety disorders: implications for novel therapeutics. American Journal of Medical Genetics Part C: Seminars in Medical Genetics; 2008.

36. Swanson LW, Sawchenko PE, Rivier J, Vale WW. Organization of ovine corticotropin-releasing factor immunoreactive cells and fibers in the rat brain: an immunohistochemical study. Neuroendocrinology. 1983; 36(3): $165-86$.

37. Chen Y, Bender RA, Frotscher M, Baram TZ. Novel and transient populations of corticotropinreleasing hormone-expressing neurons in developing hippocampus suggest unique functional roles: a quantitative spatiotemporal analysis. J Neurosci. 2001; 21(18): 7171-81

38. Valentino RJ, Van Bockstaele E. Convergent regulation of locus coeruleus activity as an adaptive response to stress. Eur J Pharmacol. 2008; 583(2-3): 194 203.

39. Gallagher JP, Orozco-Cabal LF, Liu J, ShinnickGallagher P. Synaptic physiology of central CRH system. Eur J Pharmacol. 2008; 583(2-3): 215-25.

40. Roozendaal B, Brunson KL, Holloway BL, McGaugh JL, Baram TZ. Involvement of stress-released corticotropin-releasing hormone in the basolateral amygdala in regulating memory consolidation. Proc Natl Acad Sci U S A. 2002; 99(21): 13908-13.

41. Chen Y, Brunson K, Adelmann G, Bender R, Frotscher M, Baram T. Hippocampal corticotropin releasing hormone: pre-and postsynaptic location and release by stress. Neuroscience. 2004; 126(3): 533-40.

42. Chen Y, Dubé CM, Rice CJ, Baram TZ. Rapid loss of dendritic spines after stress involves derangement of spine dynamics by corticotropin-releasing hormone. J Neurosci. 2008; 28(11): 2903-11.

43. Young EA, Abelson J, Lightman SL. Cortisol pulsatility and its role in stress regulation and health. Front Neuroendocrinol. 2004; 25(2): 69-76.

44. Meijer OC, Buurstede J, Schaaf MJ. Corticosteroid receptors in the brain: transcriptional mechanisms for specificity and context-dependent effects. Cell Mol Neurobiol. 2019; 39(4): 539-49.

45. De Kloet ER, Joëls M, Holsboer F. Stress and the brain: from adaptation to disease. Nat Rev Neurosci. 2005; 6(6): 463-75.

46. Kim JJ, Diamond DM. The stressed hippocampus, synaptic plasticity and lost memories. Nat Rev Neurosci. 2002; 3(6): 453-62.

47. Barker DJ. The foetal and infant origins of inequalities in health in Britain. J Public Health Med. 1991; 13(2): 64-8.

48. Weaver IC, Cervoni N, Champagne FA, D'Alessio 


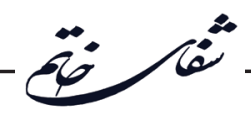

AC, Sharma S, Seckl JR, et al. Epigenetic programming by maternal behavior. Nat Neurosci. 2004; 7(8): 847-54.

49. Uno H, Lohmiller L, Thieme C, Kemnitz JW, Engle MJ, Roecker EB, et al. Brain damage induced by prenatal exposure to dexamethasone in fetal rhesus macaques. I. Hippocampus. Brain Res Dev Brain Res. 1990; 53(2): 157-67.

50. Cratty MS, Ward HE, Johnson EA, Azzaro AJ, Birkle DL. Prenatal stress increases corticotropin-releasing factor (CRF) content and release in rat amygdala minces. Brain Res. 1995; 675(1-2): 297-302.

51. Sun H, Wu H, Liu J, Wen J, Zhu Z, Li H. Prenatal stress impairs spatial learning and memory associated with lower mRNA level of the CAMKII and CREB in the adult female rat hippocampus. Neurochem Res. 2017; 42(5): 1496-503.

52. Deminière JM, Piazza PV, Guegan G, Abrous N, Maccari S, Le Moal M, et al. Increased locomotor response to novelty and propensity to intravenous amphetamine self-administration in adult offspring of stressed mothers. Brain Res. 1992; 586(1): 135-9.

53. Vallee M, Mayo W, Dellu F, Le Moal M, Simon $\mathrm{H}$, Maccari S. Prenatal stress induces high anxiety and postnatal handling induces low anxiety in adult offspring: correlation with stress-induced corticos terone secretion. J Neurosci. 1997; 17(7): 2626-36.

54. Lemaire V, Koehl M, Le Moal M, Abrous DN. Prenatal stress produces learning deficits associated with an inhibition of neurogenesis in the hippocampus. Proc Natl Acad Sci U S A. 2000; 97(20): 11032-7.

55. Piazza PV, Le Moal M. Pathophysiological basis of vulnerability to drug abuse: role of an interaction between stress, glucocorticoids, and dopaminergic neurons. Annu Rev Pharmacol Toxicol. 1996; 36(1): 359-78.

56. Wang JX, Furukawa H. Dissecting diverse functions of NMDA receptors by structural biology. Current Opinion in Structural Biology. 2019; 54: 34-42.

57. Abraham WC, Williams JM. LTP maintenance and its protein synthesis-dependence. Neurobiol Learn Mem. 2008; 89(3): 260-8.

58. Takeuchi T, Duszkiewicz AJ, Morris RG. The synaptic plasticity and memory hypothesis: encoding, storage and persistence. Philos Trans R Soc Lond B Biol Sci. 2014; 369(1633): 20130288.

59. Malinow R. AMPA receptor trafficking and longterm potentiation. Philos Trans R Soc Lond B Biol Sci.
2003; 358(1432): 707-14.

60. Huang X, Koutcherov I, Lin S, Wang H, Storlien L. Localization of leptin receptor mRNA expression in mouse brain. Neuroreport. 1996; 7(15-17): 2635-8.

61. Lynch MA. Long-term potentiation and memory. Physiol Rev. 2004; 84(1): 87-136.

62. Laplante DP, Brunet A, Schmitz N, Ciampi A, King S. Project Ice storm: prenatal maternal stress affects cognitive and linguistic functioning in $5^{11} \frac{1}{2}$-year-old children. Journal of the American Academy of Child \& Adolescent Psychiatry. 2008; 47(9): 1063-72.

63. Laplante DP, Barr RG, Brunet A, Du Fort GG, Meaney ML, Saucier J-F, et al. Stress during pregnancy affects general intellectual and language functioning in human toddlers. Pediatr Res. 2004; 56(3): 400-10.

64. DiPietro JA, Novak MF, Costigan KA, Atella LD, Reusing SP. Maternal psychological distress during pregnancy in relation to child development at age two. Child Dev. 2006; 77(3): 573-87.

65. Buss C, Davis EP, Muftuler LT, Head K, Sandman CA. High pregnancy anxiety during mid-gestation is associated with decreased gray matter density in 6â€"9year-old children. Psychoneuroendocrinology. 2010; 35(1): 141-53.

66. Yang J, Han H, Cao J, Li L, Xu L. Prenatal stress modifies hippocampal synaptic plasticity and spatial learning in young rat offspring. Hippocampus. 2006; 16(5): 431-6.

67. Del Cerro M, Perez-Laso C, Ortega E, Martín J, Gomez F, Perez-Izquierdo M, et al. Maternal care counteracts behavioral effects of prenatal environmental stress in female rats. Behav Brain Res. 2010; 208(2): 593-602.

68. Bruel-Jungerman E, Rampon C, Laroche S. Adult hippocampal neurogenesis, synaptic plasticity and memory: facts and hypotheses. Rev Neurosci. 2007; 18(2): 93-114.

69. Snyder J, Hong N, McDonald R, Wojtowicz J. A role for adult neurogenesis in spatial long-term memory. Neuroscience. 2005; 130(4): 843-52.

70. Bustamante C, Bilbao P, Contreras W, Martínez M, Mendoza A, Reyes Á, et al. Effects of prenatal stress and exercise on dentate granule cells maturation and spatial memory in adolescent mice. Int J Dev Neurosci. 2010; 28(7): 605-9.

71. Wu J, Song T-B, Li Y-J, He K-S, Ge L, Wang L-R. 
Prenatal restraint stress impairs learning and memory and hippocampal PKCbeta1 expression and translocation in offspring rats. Brain Res. 2007; 1141: 205-13.

72. Zarrow M, Philpott J, Denenberg V. Passage of $14 \mathrm{C}$-4-corticosterone from the rat mother to the foetus and neonate. Nature. 1970; 226(5250): 1058-9.

73. Boero G, Pisu MG, Biggio F, Muredda L, Carta $\mathrm{G}$, Banni $\mathrm{S}$, et al. Impaired glucocorticoid-mediated HPA axis negative feedback induced by juvenile social isolation in male rats. Neuropharmacology. 2018; 133: 242-53.

74. Sapolsky RM, Krey LC, McEwen BS. Corticosterone receptors decline in a site-specific manner in the aged rat brain. Brain Res. 1983; 289(1-2): 235-40.

75. Yao Y-Y, Wu Q-S, Li W-Z, Li W-P. Dexamethasone potentiated Abeta-induced learning and memory impairment in rats. Neurol Res. 2011; 33(4): 371-80.

76. Fujioka T, Fujioka A, Tan N, Chowdhury G, Mouri $H$, Sakata $Y$, et al. Mild prenatal stress enhances learning performance in the non-adopted rat offspring. Neuroscience. 2001; 103(2): 301-7.

77. Cannizzaro C, Plescia F, Martire M, Gagliano M, Cannizzaro G, Mantia G, et al. Single, intense prenatal stress decreases emotionality and enhances learning performance in the adolescent rat offspring: interaction with a brief, daily maternal separation. Behav Brain Res. 2006; 169(1): 128-36.

78. Salehi B, Cordero MI, Sandi C. Learning under stress: the inverted-U-shape function revisited. Learn Mem. 17(10): 522-30.

79. Bernhardt L, Bairy K, Madhyastha S. Neuroprotective role of $\mathrm{N}$-acetylcysteine against Learning deficits and Altered brain neurotransmitters in rat pups subjected to prenatal stress. Brain Sci. 2018; 8(7): 120. doi: 10.3390/ brainsci8070120.

80. Yeh CM, Huang CC, Hsu KS. Prenatal stress alters hippocampal synaptic plasticity in young rat offspring through preventing the proteolytic conversion of probrain-derived neurotrophic factor (BDNF) to mature BDNF. J Physiol. 2012; 590(4): 991-1010.

81. Son GH, Geum D, Chung S, Kim EJ, Jo J-H, Kim $\mathrm{C}-\mathrm{M}$, et al. Maternal stress produces learning deficits associated with impairment of NMDA receptormediated synaptic plasticity. J Neurosci. 2006; 26(12): 3309-18.

82. Gerges N, Aleisa A, Schwarz L, Alkadhi K. Reduced basal CAMKII levels in hippocampal CA1 region:
Possible cause of stress-induced impairment of LTP in chronically stressed rats. Hippocampus. 2004; 14(3): 402-10.

83. Van den Hove D, Steinbusch H, Scheepens A, Van de Berg W, Kooiman L, Boosten B, et al. Prenatal stress and neonatal rat brain development. Neuroscience. 2006; 137(1): 145-55.

84. Maggio N, Segal M. Striking variations in corticosteroid modulation of long-term potentiation along the septo temporal axis of the hippocampus. J Neurosci. 2007; 27(21): 5757-65.

85. Weinstock M. Prenatal stressors in rodents: Effects on behavior. Neurobiol Stress. 2017; 6: 3-13.

86. Zagron G, Weinstock M. Maternal adrenal hormone secretion mediates behavioural alterations induced by prenatal stress in male and female rats. Behav Brain Res. 2006; 175(2): 323-8.

87. Zuena $\mathrm{AR}$, Mairesse $\mathrm{J}$, Casolini $\mathrm{P}$, Cinque $\mathrm{C}$, Alemà GS, Morley-Fletcher S, et al. Prenatal restraint stress generates two distinct behavioral and neurochemical profiles in male and female rats. PloS One. 2008; 3(5): e2170.

88. Schulz KM, Pearson JN, Neeley EW, Berger R, Leonard S, Adams CE, et al. Maternal stress during pregnancy causes sex-specific alterations in offspring memory performance, social interactions, indices of anxiety, and body mass. Physiol Behav. 2011; 104(2): 340-7.

89. Zhao D, Liu D, Chen X, Wang K, Zhang A, Kang J, et al. Prenatal stress disturbs hippocampal KIF17 and NR2B in spatial cognition in male offspring. J Neurosci Res. 2013; 91(4): 535-44.

90. Weinstock M. Sex-dependent changes induced by prenatal stress in cortical and hippocampal morphology and behaviour in rats: an update. Stress. 2011; 14(6): 604-13.

91. Liu F, Day M, Muniz LC, Bitran D, Arias R, RevillaSanchez R, et al. Activation of estrogen receptor- ${ }^{2} \dot{C}$ regulates hippocampal synaptic plasticity and improves memory. Nat Neurosci. 2008; 11(3): 334-43.

92. Newhouse P, Newhouse C, Astur RS. Sex differences in visual-spatial learning using a virtual water maze in pre-pubertal children. Behav Brain Res. 2007; 183(1): $1-7$.

93. de Bruin JP, Moita MP, de Brabander HM, Joosten RN. Place and response learning of rats in a Morris water maze: differential effects of fimbria fornix and medial 


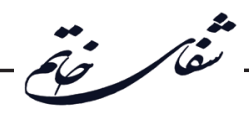

prefrontal cortex lesions. Neurobiol Learn Mem. 2001; 75(2): 164-78.

94. Blokland A, Rutten K, Prickaerts J. Analysis of spatial orientation strategies of male and female Wistar rats in a Morris water escape task. Behav Brain Res. 2006; 171(2): 216-24.

95. Bowman RE, Maclusky NJ, Sarmiento Y, Frankfurt M, Gordon M, Luine VN. Sexually dimorphic effects of prenatal stress on cognition, hormonal responses, and central neurotransmitters. Endocrinology. 2004; 145(8): 3778-87.

96. Bock J, Wainstock T, Braun K, Segal M. Stress in utero: prenatal programming of brain plasticity and cognition. Biol Psychiatry. 2015; 78(5): 315-26.

97. Yaka R, Salomon S, Matzner H, Weinstock M. Effect of varied gestational stress on acquisition of spatial memory, hippocampal LTP and synaptic proteins in juvenile male rats. Behav Brain Res. 2007; 179(1): 126-32.

98. Modir F, Salmani ME, Goudarzi I, Lashkarboluki T, Abrari K. Prenatal stress decreases spatial learning and memory retrieval of the adult male offspring of rats. Physiology \& behavior. 129: 104-9.

99. Mandyam CD, Crawford EF, Eisch AJ, Rivier CL, Richardson HN. Stress experienced in utero reduces sexual dichotomies in neurogenesis, microenvironment, and cell death in the adult rat hippocampus. Dev Neurobiol. 2008; 68(5): 575-89.

100. Hosseini-Sharifabad M, Hadinedoushan H. Prenatal stress induces learning deficits and is associated with a decrease in granules and CA3 cell dendritic tree size in rat hippocampus. Anat Sci Int. 2007; 82(4): 211-7.

101. Martínez-Téllez RI, Hernández-Torres E, Gamboa C, Flores G. Prenatal stress alters spine density and dendritic length of nucleus accumbens and hippocampus neurons in rat offspring. Synapse. 2009; 63(9): 794-804.

102. Suenaga T, Yukie M, Gao S, Nakahara D. Sex-specific effects of prenatal stress on neuronal development in the medial prefrontal cortex and the hippocampus. Neuroreport. 2012; 23(7): 430-5.

103. Konkle AT, McCarthy MM. Developmental time course of estradiol, testosterone, and dihydrotestosterone levels in discrete regions of male and female rat brain. Endocrinology. 2010; 152(1): 223-35.

104. Madeira MD, Lieberman AR. Sexual dimorphism in the mammalian limbic system. Prog Neurobiol. 1995; 45(4): 275-333.
105. Pedersen BK. Physical activity and muscle-brain crosstalk. Nature Reviews Endocrinology. 2019; 15: 383-92.

106. Ezmerli NM. Exercise in pregnancy. Primary Care Update for Ob/Gyns. 2000; 7(6): 260-5.

107. Gomes da Silva S, Arida RM. Physical activity and brain development. Expert Review of Neurotherapeutics. 2015; 15(9): 1041-51.

108. Akhavan MM, Miladi-Gorji H, Emami-Abarghoie M, Safari M, Sadighi-Moghaddam B, Vafaei AA, et al. Maternal voluntary exercise during pregnancy enhances the spatial learning acquisition but not the retention of memory in rat pups via a TrkB-mediated mechanism: the role of hippocampal BDNF Expression. Iran J Basic Med Sci. 2013; 16(9): 955-61.

109. Kim D-M, Leem Y-H. Chronic stress-induced memory deficits are reversed by regular exercise via AMPK-mediated BDNF induction. Neuroscience. 2016; 324: 271-85.

110. Schmitt U, Tanimoto N, Seeliger M, Schaeffel F, Leube R. Detection of behavioral alterations and learning deficits in mice lacking synaptophysin. Neuroscience. 2009; 162(2): 234-43.

111. El-Husseini AE-D, Schnell E, Chetkovich DM, Nicoll RA, Bredt DS. PSD-95 involvement in maturation of excitatory synapses. Science. 2000; 290(5495): 1364-8.

112. Kim T-W, Shin M-S, Park J-K, Shin M-A, Lee H-H, Lee S-J. Treadmill exercise alleviates prenatal noise stress-induced impairment of spatial learning ability through enhancing hippocampal neurogenesis in rat pups. J Exerc Rehabil. 2013; 9(5): 451-6.

113. Zubedat S, Aga-Mizrachi S, Cymerblit-Sabba A, Ritter A, Nachmani M, Avital A. Methylphenidate and environmental enrichment ameliorate the deleterious effects of prenatal stress on attention functioning. Stress. 2015; 18(3): 280-8.

114. Ohline SM, Abraham WC. Environmental enrichment effects on synaptic and cellular physiology of hippocampal neurons. Neuropharmacology. 2019; 145(Pt A): 3-12.

115. Vazquez-Sanroman D, Sanchis-Segura C, Toledo R, Hernández ME, Manzo J, Miquel M. The effects of enriched environment on BDNF expression in the mouse cerebellum depending on the length of exposure. Behavioural Brain Research. 2013; 243: 118-28.

116. Li M, Wang M, Ding S, Li C, Luo X. Environmental 
enrichment during gestation improves behavior consequences and synaptic plasticity in hippocampus of prenatal-stressed offspring rats. Acta Histochem Cytochem. 2012; 45(3): 157-66.

117. Morley-Fletcher S, Rea M, Maccari S, Laviola G. Environmental enrichment during adolescence reverses the effects of prenatal stress on play behaviour and HPA axis reactivity in rats. European Journal of Neuroscience. 2003; 18(12): 3367-74.

118. Zhang Z, Zhang H, Du B, Chen Z. Neonatal handling and environmental enrichment increase the expression of GAP-43 in the hippocampus and promote cognitive abilities in prenatally stressed rat offspring. Neuroscience Letters. 2012; 522(1): 1-5.

119. Shefa U, Jeong NY, Song IO, Chung HJ, Kim D, Jung J, et al. Mitophagy links oxidative stress conditions and neurodegenerative diseases. Neural Regen Res. 2019; 14(5): 749-56.

120. Toma VA, Farcas AD, Parvu M, Silaghi-Dumitrescu R, Roman I. CA3 hippocampal field: Cellular changes and its relation with blood nitro-oxidative stress reveal a balancing function of $\mathrm{CA} 3$ area in rats exposed to repetead restraint stress. Brain Research Bulletin. 2017; 130: $10-7$.

121. Alzoubi KH, Khabour OF, Rashid BA, Damaj IM, Salah HA. The neuroprotective effect of vitamin E on chronic sleep deprivation-induced memory impairment: the role of oxidative stress. Behavioural Brain Research. 2012; 226(1): 205-10.

122. Alqudah MAY, Alzoubi KH, Ma'abrih GM, Khabour OF. Vitamin C prevents memory impairment induced by waterpipe smoke: role of oxidative stress. Inhalation Toxicology. 2018; 30(4-5): 141-8.

123. Alzoubi KH, Rababa'h AM, Owaisi A, Khabour OF. L-carnitine prevents memory impairment induced by chronic REM-sleep deprivation. Brain Res Bull. 2017; 131: 176-82.

124. Mohammadi HS, Goudarzi I, Lashkarbolouki T, Abrari K, Elahdadi Salmani M. Chronic administration of quercetin prevent spatial learning and memory deficits provoked by chronic stress in rats. Behav Brain Res. 2014; 270: 196-205. 